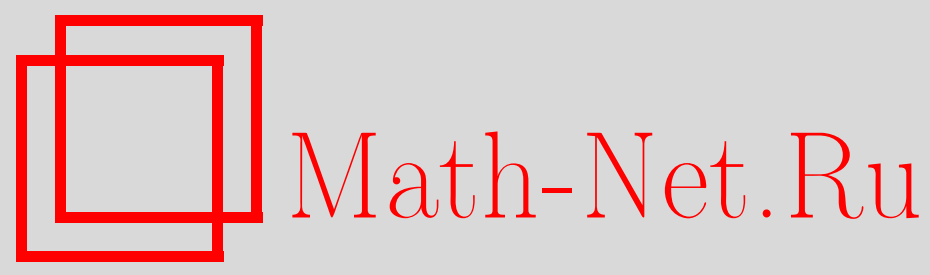

П. Л. Гуревич, Разрешимость нелокальных эллиптических задач в двугранных углах, Матем. заметки, 2002, том 72, выпуск 2, 178-197

DOI: https://doi.org/10.4213/mzm413

Использование Общероссийского математического портала Math-Net.Ru подразумевает, что вы прочитали и согласны с пользовательским соглашением http://www . mathnet.ru/rus/agreement

Параметры загрузки:

IP : 54.164 .48 .24

26 апреля 2023 г., 13:49:26

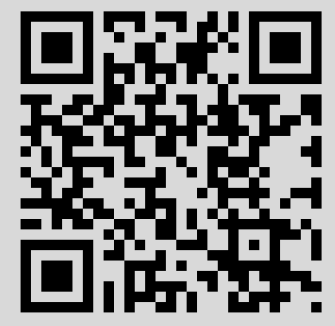




\title{
РАЗРЕШИМОСТЬ НЕЛОКАЛЬНЫХ ЭЛЛИПТИЧЕСКИХ ЗАДАЧ В ДВУГРАННЫХ УГЛАХ
}

П. Л. Гуревич

\begin{abstract}
В работе рассматриваются нелокальные эллиптические задачи в двугранных и плоских углах, возникающие при изучении нелокальных задач в ограниченной области в случае пересечения носителя нелокальных данных с границей. Изучаются фредгольмова и однозначная разрешимость указанных задач в соответствующих весовых пространствах. В качестве аппарата исследований применяются априорные оценки решений и формула Грина для нелокальных эллиптических задач.

Библиографоия: 15 названий.
\end{abstract}

При изучении эллиптических задач с нелокальньми условиями наибольшие трудности возникают в случае пересечения носителя нелокальных данных с границей (см. [1]-[4]). Это приводит к появлению степенных особенностей у решений вблизи некоторого множества, поэтому нелокальные эллиптические задачи естественно рассматривать в весовых пространствах (см. [5]-[7]). При получении априорных оценок решений и построении правого регуляризатора нелокальных задач в ограниченной области возникают модельные нелокальные краевые задачи в двугранных углах (см. [3], [4]). В настоящей работе предлагается подход к изучению нелокальных задач, основанный на использовании формулы Грина и сопряженных нелокальных задач. Такой подход позволяет снять дополнительные ограничения работы [3] на соответствующую "локальную" модельную задачу и получить необходимые и достаточные условия фредгольмовости нелокальных задач в плоских углах и однозначной разрешимости таких задач в двугранных углах. При этом в качестве сопряженных возникают нелокальные задачи трансмиссии, рассматривавшиеся в работах [8] (в случае ограниченной области с гладкой границей), [9] (в одномерном случае) и др.

В данной работе мы ограничимся для наглядности нелокальными возмущениями задачи Дирихле для оператора Лапласа.

\section{1. Постановка нелокальных эллиптических краевых задач}

1.1. Введем двугранньй угол

$$
\Omega=\left\{x=(y, z): r>0, b_{1}<\varphi<b_{2}, z \in \mathbb{R}^{n-2}\right\}
$$

Работа выполнена при финансовой поддержке Российского фонда фундаментальных исследований, грант № 01-01-01030, а также гранта № Е00-1-195 Министерства образования РФ. 
с гранями

$$
\Gamma_{j}=\left\{x=(y, z): r>0, \varphi=b_{j}, z \in \mathbb{R}^{n-2}\right\}, \quad j=1,2,
$$

и ребром $M=\left\{x=(y, z): y=0, z \in \mathbb{R}^{n-2}\right\}$. Здесь $x=(y, z) \in \mathbb{R}^{n}, y \in \mathbb{R}^{2}, z \in \mathbb{R}^{n-2}$; $r, \varphi$ - полярные координаты точки $y ; 0<b_{1}<b_{2}<2 \pi$. Рассмотрим нелокальную краевую задачу в двугранном угле $\Omega$

$$
\begin{gathered}
\Delta U(x)=f(x), \quad x \in \Omega, \\
\left.U(x)\right|_{\Gamma_{j}}+\left.a_{j} U\left(\mathscr{G}_{j} y, z\right)\right|_{\Gamma_{j}}=g_{j}(x), \quad x \in \Gamma_{j} .
\end{gathered}
$$

Здесь и далее индекс $j$ принимает значения $j=1,2 ; a_{j} \in \mathbb{C} ; \mathscr{G}_{j}$ есть оператор поворота на угол $\varphi_{j}$ и растяжения в $\chi_{j}$ раз в плоскости $\{y\}$. При этом $b_{1}<b_{1}+\varphi_{1}=b_{2}+\varphi_{2}=$ $b<b_{2}, 0<\chi_{j}$.

Введем пространство $H_{a}^{l}(\Omega)$ как пополнение множества $C_{0}^{\infty}(\bar{\Omega} \backslash M)$ по норме

$$
\|w\|_{H_{a}^{l}(\Omega)}=\left(\sum_{|\alpha| \leqslant l} \int_{\Omega} r^{2(a-l+|\alpha|)}\left|D_{x}^{\alpha} w(x)\right|^{2} d x\right)^{1 / 2}
$$

где $C_{0}^{\infty}(\bar{\Omega} \backslash M)$ - множество бесконечно дифференцируемых в $\bar{\Omega}$ функций с компактными носителями из $\bar{\Omega} \backslash M ; a \in \mathbb{R}, l \geqslant 0$ целое. Через $H_{a}^{l-1 / 2}\left(\Gamma^{\prime}\right)$ при $l \geqslant 1$ обозначим пространство следов на $\Gamma^{\prime}=\left\{x=(y, z): r>0, \varphi=b^{\prime}, z \in \mathbb{R}^{n-2}\right\}, b_{1} \leqslant b^{\prime} \leqslant b_{2}$, с нормой $\|\psi\|_{H_{a}^{l-1 / 2}\left(\Gamma^{\prime}\right)}=\inf \|w\|_{H_{a}^{l}(\Omega)}, w \in H_{a}^{l}(\Omega):\left.w\right|_{\Gamma^{\prime}}=\psi$. Введем ограниченный оператор

$$
\mathscr{L}_{\Omega}: H_{a}^{2}(\Omega) \rightarrow H_{a}^{0}(\Omega, \Gamma)=H_{a}^{0}(\Omega) \times \prod_{j} H_{a}^{3 / 2}\left(\Gamma_{j}\right)
$$

по формуле

$$
\mathscr{L}_{\Omega} U=\left\{\Delta U,\left.U(x)\right|_{\Gamma_{j}}+\left.a_{j} U\left(\mathscr{G}_{j} y, z\right)\right|_{\Gamma_{j}}\right\} .
$$

Обозначим через $W^{l}(Q), l \geqslant 0$ целое, пространство Соболева функций, интегрируемых в квадрате вместе со всеми обобщенными производньми вплоть до $l$-го порядка по $Q$, где $Q \subset \mathbb{R}^{n}$ - область с липшицевой границей. Через $W^{l-1 / 2}(\Upsilon)$ при $l \geqslant 1$ обозначим пространство следов на $(n-1)$-мерном гладком многообразии $\Upsilon \subset \bar{Q}$.

Лемма 1.1. Для любьх $w \in W^{l}(Q) u \lambda \in \mathbb{C}$ имеет место оиенка

$$
|\lambda|^{l-s}\|w\|_{W^{s}(Q)} \leqslant c\left(\|w\|_{W^{l}(Q)}+|\lambda|^{l}\|w\|_{L_{2}(Q)}\right)
$$

Здесь $0<s<l ; c>0$ не зависит от $w, \lambda$.

Лемма 1.2. Для любых $w \in W^{1}(Q)$ и $\lambda \in \mathbb{C}$ выполнена оченка

$$
|\lambda|^{1 / 2}\left\|\left.w\right|_{\Upsilon}\right\|_{L^{2}(\Upsilon)} \leqslant c\left(\|w\|_{W^{1}(Q)}+|\lambda|\|w\|_{L_{2}(Q)}\right) .
$$

Здесь $с>0$ не зависит от $w, \lambda$.

Доказательство лемм 1.1, 1.2 содержится в [10,гл. 1]. Используя лемму 1.1 и свойства весовых пространств, в работе $[2, \S 1]$ был получен следующий результат. 
Лемма 1.3. Для любих $w \in H_{a}^{l}(\Omega) u \lambda \in \mathbb{C}$ выполнено

$$
|\lambda|^{s}\|w\|_{H_{a-s}^{l-s}(\Omega)} \leqslant c\left(\|w\|_{H_{a}^{l}(\Omega)}+|\lambda|^{l}\|w\|_{H_{a-l}^{0}(\Omega)}\right) .
$$

Здесь $0<s<l ; c>0$ не зависит от $w, \lambda$.

1.2. Рассмотрим вспомогательную нелокальную краевую задачу в плоском угле:

$$
\begin{gathered}
\Delta u(y)-u(y)=f(y), \quad y \in K, \\
\left.u(y)\right|_{\gamma_{j}}+\left.a_{j} u\left(\mathscr{G}_{j} y\right)\right|_{\gamma_{j}}=g_{j}(y), \quad y \in \gamma_{j},
\end{gathered}
$$

где $K=\left\{y \in \mathbb{R}^{2}: r>0,0<b_{1}<\varphi<b_{2}<2 \pi\right\}, \gamma_{j}=\left\{y \in \mathbb{R}^{2}: r>0, \varphi=b_{j}\right\}$.

Аналогично предыдущему вводятся пространства функций $H_{a}^{l}(K)$ и $H_{a}^{l-1 / 2}\left(\gamma^{\prime}\right)$, где $\gamma^{\prime}=\left\{y \in \mathbb{R}^{2}: r>0, \varphi=b^{\prime}\right\}, b_{1} \leqslant b^{\prime} \leqslant b_{2}$. Введем пространство $E_{a}^{l}(K)$ как пополнение $C_{0}^{\infty}(\bar{K} \backslash\{0\})$ по норме

$$
\|w\|_{E_{a}^{l}(K)}=\left(\sum_{|\alpha| \leqslant l} \int_{K} r^{2 a}\left(r^{2(|\alpha|-l)}+1\right)\left|D_{y}^{\alpha} w(y)\right|^{2} d y\right)^{1 / 2} .
$$

Через $E_{a}^{l-1 / 2}\left(\gamma^{\prime}\right)$ при $l \geqslant 1$ обозначим пространство следов на луче $\gamma^{\prime}$ с нормой

$$
\|\psi\|_{E_{a}^{l-1 / 2}\left(\gamma^{\prime}\right)}=\inf \|w\|_{E_{a}^{l}(K)}, \quad w \in E_{a}^{l}(K):\left.w\right|_{\gamma^{\prime}}=\psi
$$

Конструктивные определения пространств $H_{a}^{l-1 / 2}\left(\Gamma^{\prime}\right), H_{a}^{l-1 / 2}\left(\gamma^{\prime}\right)$ и $E_{a}^{l-1 / 2}\left(\gamma^{\prime}\right)$, эквивалентные данным, см. в $[6, \S 1]$. Установим одно свойство весовых пространств, которое потребуется ниже.

ЛЕмма 1.4. Для любой $\psi \in E_{a}^{l-1 / 2}\left(\gamma^{\prime}\right)$ имеет место оценка

$$
\left(\int_{\gamma^{\prime}} r^{2(a-(l-1 / 2))}|\psi|^{2} d \gamma\right)^{1 / 2} \leqslant c\|\psi\|_{E_{a}^{l-1 / 2}\left(\gamma^{\prime}\right)},
$$

әде с >0 не зависит от $\psi$.

ДоКАЗАТЕЛЬСТВо. Из $[7$, гл. 6, п. 1.3$]$ следует, что норма $\|u\|_{E_{a}^{l}(K)}$ и норма

$$
\left(\sum_{k=0}^{l} \int_{0}^{\infty} r^{2(a-(l-1 / 2))} \sum_{j=0}^{l-k}(1+r)^{2(l-k-j)}\left\|\left(r D_{r}\right)^{k} u(r, \cdot)\right\|_{W^{j}\left(b_{1}, b_{2}\right)}^{2} d r\right)^{1 / 2}
$$

эквивалентны; здесь $u(r, \varphi)$ есть функция $u(y)$, записанная в полярных координатах.

Выберем функцию $u \in E_{a}^{l}(K)$ так, что $\left.u\right|_{\gamma^{\prime}}=\psi,\|u\|_{E_{a}^{l}(K)} \leqslant 2\|\psi\|_{E_{a}^{l-1 / 2}\left(\gamma^{\prime}\right)}$. Поскольку $\left.u(r, \varphi)\right|_{\varphi=b^{\prime}}=\psi(r)$, в силу непрерьвности операции взятия следа в пространствах Соболева имеем $|\psi(r)|^{2} \leqslant k_{1}\|u(r, \cdot)\|_{W^{l}\left(b_{1}, b_{2}\right)}^{2}$. Отсюда и из эквивалентности нормы $\|u\|_{E_{a}^{l}(K)}$ и нормы (1.8) получим

$$
\int_{\gamma^{\prime}} r^{2(a-(l-1 / 2))}|\psi|^{2} d \gamma \leqslant k_{1} \int_{0}^{\infty} r^{2(a-(l-1 / 2))}\|u(r, \cdot)\|_{W^{l}\left(b_{1}, b_{2}\right)}^{2} d r \leqslant k_{2}\|u\|_{E_{a}^{l}(K)}^{2} .
$$


Из (1.9) и неравенства $\|u\|_{E_{a}^{l}(K)} \leqslant 2\|\psi\|_{E_{a}^{l-1 / 2}\left(\gamma^{\prime}\right)}$ следует утверждение леммы.

Введем ограниченньй оператор

$$
\mathscr{L}_{K}: E_{a}^{2}(K) \rightarrow E_{a}^{0}(K, \gamma)=E_{a}^{0}(K) \times \prod_{j} E_{a}^{3 / 2}\left(\gamma_{j}\right)
$$

по формуле

$$
\mathscr{L}_{K} u=\left\{\Delta u-u,\left.u(y)\right|_{\gamma_{j}}+\left.a_{j} u\left(\mathscr{G}_{j} y\right)\right|_{\gamma_{j}}\right\} .
$$

1.3. Следуя работе [3], рассмотрим модельную аналитическую оператор-функцию

$$
\widetilde{\mathscr{L}}(\lambda): W^{2}\left(b_{1}, b_{2}\right) \rightarrow W^{0}\left[b_{1}, b_{2}\right]=L_{2}\left(b_{1}, b_{2}\right) \times \mathbb{C}^{2},
$$

определенную по формуле

$$
\widetilde{\mathscr{L}}(\lambda) \widetilde{U}=\left\{\frac{d^{2}}{d \varphi^{2}} \widetilde{U}(\varphi)-\lambda^{2} \widetilde{U}(\varphi),\left.\quad \widetilde{U}(\varphi)\right|_{\varphi=b_{j}}+\left.a_{j} e^{i \lambda \ln \chi_{j}} \widetilde{U}\left(\varphi+\varphi_{j}\right)\right|_{\varphi=b_{j}}\right\} .
$$

Введем в гильбертовых пространствах $W^{2}\left(b_{1}, b_{2}\right)$ и $W^{0}\left[b_{1}, b_{2}\right]$ эквивалентные нормы, зависящие от параметра $\lambda \in \mathbb{C}(|\lambda| \geqslant 1)$ :

$$
\begin{gathered}
\|\widetilde{U}\|_{W^{2}\left(b_{1}, b_{2}\right)}=\left(\|\widetilde{U}\|_{W^{2}\left(b_{1}, b_{2}\right)}^{2}+|\lambda|^{4}\|\widetilde{U}\|_{L_{2}\left(b_{1}, b_{2}\right)}^{2}\right)^{1 / 2}, \\
\|\|\left\{\widetilde{F}, \widetilde{G}_{j}\right\}\|\|_{W^{0}\left[b_{1}, b_{2}\right]}=\left(\|\widetilde{F}\|_{L_{2}\left(b_{1}, b_{2}\right)}^{2}+\sum_{j}|\lambda|^{3}\left|\widetilde{G}_{j}\right|^{2}\right)^{1 / 2} .
\end{gathered}
$$

Лемма 1.5. Для всех $\lambda \in \mathbb{C}$ оператор $\widetilde{\mathscr{L}}(\lambda)$ фредгольмов, ind $\widetilde{\mathscr{L}}(\lambda)=0 ;$ для любого $h \in \mathbb{R}$ сушествует $q_{0}>1$ такое, что для $\lambda \in J_{h, q_{0}}=\left\{\lambda \in \mathbb{C}: \operatorname{Im} \lambda=h,|\operatorname{Re} \lambda| \geqslant q_{0}\right\}$ оператор $\widetilde{\mathscr{L}}(\lambda)$ имеет ограниченный обратны й $\widetilde{\mathscr{L}}^{-1}(\lambda): W^{0}\left[b_{1}, b_{2}\right] \rightarrow W^{2}\left(b_{1}, b_{2}\right) u$

$$
\|\widetilde{\mathscr{L}}-1(\lambda) \widetilde{\Phi}\|_{W^{2}\left(b_{1}, b_{2}\right)} \leqslant c \mid\|\widetilde{\Phi}\|_{W^{0}\left[b_{1}, b_{2}\right]}
$$

для всех $\widetilde{\Phi} \in W^{0}\left[b_{1}, b_{2}\right]$, где с > 0 не зависит от $\lambda u \widetilde{\Phi} ;$ оператор-функиия $\widetilde{\mathscr{L}}^{-1}(\lambda)$ : $W^{0}\left[b_{1}, b_{2}\right] \rightarrow W^{2}\left(b_{1}, b_{2}\right)$ конечномероморфна.

Лемма 1.6. Для любого $0<\varepsilon<1 / \max \left|\ln \chi_{j}\right|$ существует $q>1$ такое, что множество $\{\underset{\widetilde{L}}{\lambda} \in \mathbb{C}:|\operatorname{Im} \lambda| \leqslant \varepsilon \ln |\operatorname{Re} \lambda|,|\operatorname{Re} \lambda| \geqslant q\}$ не содержит полюсов оператор-функиии $\widetilde{\mathscr{L}}^{-1}(\lambda)$; для кажсдого полюса $\lambda_{0}$ оператор-функиии $\widetilde{\mathscr{L}}^{-1}(\lambda)$ существует $\delta>0$ такое, что множество $\left\{\lambda \in \mathbb{C}: 0<\left|\operatorname{Im} \lambda-\operatorname{Im} \lambda_{0}\right|<\delta\right\}$ не содер жит полюсов оператор-функиии $\widetilde{\mathscr{L}}^{-1}(\lambda)$.

Леммы $1.5,1.6$ доказаны в $[3, \S 2]$. В $[3, \S 3]$ получен также следующий результат.

ТЕОРема 1.1. Пусть на прямой $\operatorname{Im} \lambda=a-1$ нет полюсов оператор-функиии $\widetilde{\mathscr{L}}^{-1}(\lambda)$. Тогда для всех $u \in E_{a}^{2}(K)$ выполнена оценка

$$
\|u\|_{E_{a}^{2}(K)} \leqslant c\left(\left\|\mathscr{L}_{K} u\right\|_{E_{a}^{0}(K, \gamma)}+\|u\|_{L_{2}(K \cap S)}\right),
$$

где $S=\left\{y \in \mathbb{R}^{2}: 0<R_{1}<r<R_{2}\right\} ;$ с $>0$ не зависит от и.

Если для всех $u \in E_{a}^{2}(K)$ имеет место оченка (1.11), то на прямой $\operatorname{Im} \lambda=a-1$ нет полюсов оператор-функиии $\widetilde{\mathscr{L}}^{-1}(\lambda)$.

Из теоремы 1.1 вытекает конечномерность ядра и замкнутость образа оператора $\mathscr{L}_{K}$. Для доказательства конечномерности коядра оператора $\mathscr{L}_{K}$ получим формулы Грина для нелокальных задач и изучим задачи, сопряженные к нелокальным краевьм задачам относительно формулы Грина. 


\section{2. Формулы Грина для нелокальных эллиптических задач}

2.1. Введем множество $\gamma=\left\{y \in \mathbb{R}^{2}: r>0, \varphi=b\right\}$ (напомним: $b=b_{j}+\varphi_{j}$ ). Множество $\gamma$ является носителем нелокальных данных в задаче (1.6), (1.7). Обозначим

$$
K_{1}=\left\{y \in \mathbb{R}^{2}: r>0, b_{1}<\varphi<b\right\}, \quad K_{2}=\left\{y \in \mathbb{R}^{2}: r>0, b<\varphi<b_{2}\right\} .
$$

Пусть $n_{j}$ есть нормаль к $\gamma_{j}$, направленная вне области $K_{j}, n$ - нормаль к $\gamma$, направленная вне области $K_{2}$. Будем обозначать через $(\cdot, \cdot)_{K_{j}},(\cdot, \cdot)_{\gamma_{j}},(\cdot, \cdot)_{\gamma}$ скалярные произведения в $L_{2}\left(K_{j}\right), L_{2}\left(\gamma_{j}\right), L_{2}(\gamma)$ соответственно.

Tеорема 2.1. Для $u \in C_{0}^{\infty}(\bar{K} \backslash\{0\}), v_{j} \in C^{\infty}\left(\bar{K}_{j} \backslash\{0\}\right)$ имеет место формула Грина

$$
\begin{aligned}
& \sum_{j}\left(\Delta u-u, v_{j}\right)_{K_{j}}+\sum_{j}\left(\left.u\right|_{\gamma_{j}}+\left.a_{j} u\left(\mathscr{G}_{j} y\right)\right|_{\gamma_{j}},\left.\frac{\partial v_{j}}{\partial n_{j}}\right|_{\gamma_{j}}\right)_{\gamma_{j}} \\
& \quad+\left(\left.u\right|_{\gamma},\left.\frac{\partial v_{2}}{\partial n}\right|_{\gamma}-\left.\frac{\partial v_{1}}{\partial n}\right|_{\gamma}-\left.\sum_{k} \bar{a}_{k} \chi_{k}^{-1} \frac{\partial v_{k}}{\partial n_{k}}\left(\mathscr{G}_{k}^{-1} y\right)\right|_{\gamma}\right)_{\gamma} \\
& =\sum_{j}\left(u, \Delta v_{j}-v_{j}\right)_{K_{j}}+\sum_{j}\left(\left.\frac{\partial u}{\partial n_{j}}\right|_{\gamma_{j}},\left.v_{j}\right|_{\gamma_{j}}\right)_{\gamma_{j}}+\left(\left.\frac{\partial u}{\partial n}\right|_{\gamma},\left.v_{2}\right|_{\gamma}-\left.v_{1}\right|_{\gamma}\right)_{\gamma},
\end{aligned}
$$

где $\mathscr{G}_{k}^{-1}$ - оператор поворота на угол $-\varphi_{k}$ и растяжения в $1 / \chi_{k}$ раз в плоскос$m u\{y\}$; индекс $k$ здесь и далее принимает значения $k=1,2$.

ДокАЗАТЕЛЬСтво. Умножим $\Delta u-u$ на $\bar{v}_{j}$, проинтегрируем по $K_{j}$ и воспользуемся дважды формулой интегрирования по частям; в результате получим

$$
\begin{aligned}
& \int_{K_{1}}(\Delta u-u) \cdot \bar{v}_{1} d x+\left.\left.\int_{\gamma_{1}} u\right|_{\gamma_{1}} \cdot \frac{\partial \bar{v}_{1}}{\partial n_{1}}\right|_{\gamma_{1}} d \gamma-\left.\left.\int_{\gamma} u\right|_{\gamma} \cdot \frac{\partial \bar{v}_{1}}{\partial n}\right|_{\gamma} d \gamma \\
& =\int_{K_{1}} u \cdot\left(\Delta \bar{v}_{1}-\bar{v}_{1}\right) d y+\left.\left.\int_{\gamma_{1}} \frac{\partial u}{\partial n_{1}}\right|_{\gamma_{1}} \cdot \bar{v}_{1}\right|_{\gamma_{1}} d \gamma-\left.\left.\int_{\gamma} \frac{\partial u}{\partial n}\right|_{\gamma} \cdot \bar{v}_{1}\right|_{\gamma} d \gamma \\
& \int_{K_{2}}(\Delta u-u) \cdot \bar{v}_{2} d x+\left.\left.\int_{\gamma} u\right|_{\gamma} \cdot \frac{\partial \bar{v}_{2}}{\partial n}\right|_{\gamma} d \gamma+\left.\left.\int_{\gamma_{2}} u\right|_{\gamma_{2}} \cdot \frac{\partial \bar{v}_{2}}{\partial n_{2}}\right|_{\gamma_{2}} d \gamma \\
& =\int_{K_{2}} u \cdot\left(\Delta \bar{v}_{2}-\bar{v}_{2}\right) d y+\left.\left.\int_{\gamma} \frac{\partial u}{\partial n}\right|_{\gamma} \cdot \bar{v}_{2}\right|_{\gamma} d \gamma+\left.\left.\int_{\gamma_{2}} \frac{\partial u}{\partial n_{2}}\right|_{\gamma_{2}} \cdot \bar{v}_{2}\right|_{\gamma_{2}} d \gamma .
\end{aligned}
$$

Сложим последние два равенства:

$$
\begin{aligned}
& \sum_{j} \int_{K_{j}}(\Delta u-u) \cdot \bar{v}_{j} d x+\left.\left.\sum_{j} \int_{\gamma_{j}} u\right|_{\gamma_{j}} \cdot \frac{\partial \bar{v}_{j}}{\partial n_{j}}\right|_{\gamma_{j}} d \gamma+\left.\int_{\gamma} u\right|_{\gamma} \cdot\left(\left.\frac{\partial \bar{v}_{2}}{\partial n}\right|_{\gamma}-\left.\frac{\partial \bar{v}_{1}}{\partial n}\right|_{\gamma}\right) d \gamma \\
& \quad=\sum_{j} \int_{K_{j}} u \cdot\left(\Delta \bar{v}_{j}-\bar{v}_{j}\right) d x+\left.\left.\sum_{j} \int_{\gamma_{j}} \frac{\partial u}{\partial n_{j}}\right|_{\gamma_{j}} \cdot \bar{v}_{j}\right|_{\gamma_{j}} d \gamma+\left.\int_{\gamma} \frac{\partial u}{\partial n}\right|_{\gamma} \cdot\left(\left.\bar{v}_{2}\right|_{\gamma}-\left.\bar{v}_{1}\right|_{\gamma}\right) d \gamma
\end{aligned}
$$


Ho

$$
\begin{gathered}
\left.\left.\int_{\gamma_{j}} u\right|_{\gamma_{j}} \cdot \frac{\partial \bar{v}_{j}}{\partial n_{j}}\right|_{\gamma_{j}} d \gamma=\left.\int_{\gamma_{j}}\left(\left.u\right|_{\gamma_{j}}+\left.a_{j} u\left(\mathscr{G}_{j} y\right)\right|_{\gamma_{j}}\right) \cdot \frac{\partial \bar{v}_{j}}{\partial n_{j}}\right|_{\gamma_{j}} d \gamma-\left.\left.\int_{\gamma_{j}} a_{j} u\left(\mathscr{G}_{j} y\right)\right|_{\gamma_{j}} \cdot \frac{\partial \bar{v}_{j}}{\partial n_{j}}\right|_{\gamma_{j}} d \gamma \\
=\left.\int_{\gamma_{j}}\left(\left.u\right|_{\gamma_{j}}+\left.a_{j} u\left(\mathscr{G}_{j} y\right)\right|_{\gamma_{j}}\right) \cdot \frac{\partial \bar{v}_{j}}{\partial n_{j}}\right|_{\gamma_{j}} d \gamma-\left.\left.\int_{\gamma} u\right|_{\gamma} \cdot a_{j} \chi_{j}^{-1} \frac{\partial \bar{v}_{j}}{\partial n_{j}}\left(\mathscr{G}_{j}^{-1} y\right)\right|_{\gamma} d \gamma
\end{gathered}
$$

где $\mathscr{G}_{j}^{-1}$ - оператор поворота на угол $-\varphi_{j}$ и растяжения в $1 / \chi_{j}$ раз в плоскости $\{y\}$. Отсюда и из (2.2) следует формула (2.1).

ЗАМЕчАниЕ 2.1. Формула (2.1) распространяется по непрерывности на случай $u \in$ $E_{a}^{2}(K), v_{j} \in E_{-a+2}^{2}(K)$. Действительно, $C_{0}^{\infty}(\bar{K} \backslash\{0\})$ плотно в $E_{a}^{2}(K), C_{0}^{\infty}\left(\bar{K}_{j} \backslash\{0\}\right)$ плотно в $E_{-a+2}^{2}\left(K_{j}\right)$, поэтому найдутся последовательности $\left\{u_{p}\right\}_{p=1}^{\infty} \subset C_{0}^{\infty}(\bar{K} \backslash\{0\})$ и $\left\{v_{j q}\right\}_{q=1}^{\infty} \subset C_{0}^{\infty}\left(\bar{K}_{j} \backslash\{0\}\right)$, сходящиеся к $u$ и $v$ в $E_{a}^{2}(K)$ и $E_{-a+2}^{2}\left(K_{j}\right)$ соответственно. При этом для функций $u_{p}$ и $v_{j q}$ справедлива формула Грина (2.1). Переходя к пределу при $p, q \rightarrow \infty$, получаем формулу Грина для функций $u$ и $v$ (переход к пределу возможен в силу неравенства Коши-Буняковского и леммы 1.4).

2.2. Будем обозначать через $(\cdot, \cdot)_{\beta_{1}},(\cdot, \cdot)_{\beta_{2}},(\cdot, \cdot)_{\mathbb{C}}$ скалярные произведения в $L_{2}\left(b_{1}, b\right), L_{2}\left(b, b_{2}\right), \mathbb{C}$ соответственно. Аналогично теореме 2.1 доказьвается

Теорема 2.2. Для любых $\widetilde{U} \in C^{\infty}\left(\left[b_{1}, b_{2}\right]\right), \widetilde{V}_{1} \in C^{\infty}\left(\left[b_{1}, b\right]\right), \widetilde{V}_{2} \in C^{\infty}\left(\left[b, b_{2}\right]\right) u$ $\lambda \in \mathbb{C}$ имеет место формула Грина с параметром $\lambda$ :

$$
\begin{gathered}
\sum_{j}\left(\frac{d^{2}}{d \varphi^{2}} \widetilde{U}-\lambda^{2} \widetilde{U}, \widetilde{V}_{j}\right)_{\beta_{j}}+\sum_{j}\left(\left.\widetilde{U}\right|_{\varphi=b_{j}}+\left.a_{j} e^{i \lambda \ln \chi_{j}} \widetilde{U}\left(\varphi+\varphi_{j}\right)\right|_{\varphi=b_{j}},\left.(-1)^{j} \frac{d \widetilde{V}_{j}}{d \varphi}\right|_{\varphi=b_{j}}\right)_{\mathbb{C}} \\
+\left(\left.\widetilde{U}\right|_{\varphi=b},\left.\frac{d \widetilde{V}_{1}}{d \varphi}\right|_{\varphi=b}-\left.\frac{d \widetilde{V}_{2}}{d \varphi}\right|_{\varphi=b}-\left.\sum_{k}(-1)^{k} \bar{a}_{k} e^{-i \bar{\lambda} \ln \chi_{k}} \frac{d \widetilde{V}_{k}}{d \varphi}\left(\varphi-\varphi_{k}\right)\right|_{\varphi=b}\right)_{\mathbb{C}} \\
=\sum_{j}\left(\widetilde{U}, \frac{d^{2}}{d \varphi^{2}} \widetilde{V}_{j}-\bar{\lambda}^{2} \widetilde{V}_{j}\right)_{\beta_{j}}+\sum_{j}\left(\left.(-1)^{j} \frac{d \widetilde{U}}{d \varphi}\right|_{\varphi=b_{j}},\left.\widetilde{V}_{j}\right|_{\varphi=b_{j}}\right)_{\mathbb{C}} \\
+\left(-\left.\frac{d \widetilde{U}}{d \varphi}\right|_{\varphi=b},\left.\widetilde{V}_{2}\right|_{\varphi=b}-\left.\widetilde{V}_{1}\right|_{\varphi=b}\right)_{\mathbb{C}}
\end{gathered}
$$

ЗАмЕчАниЕ 2.2. Формула (2.3) распространяется по непрерьвности на случай $\widetilde{U} \in$ $W^{2}\left(b_{1}, b_{2}\right), \widetilde{V}_{1} \in W^{2}\left(b_{1}, b\right), \widetilde{V}_{2} \in W^{2}\left(b, b_{2}\right)$ (см. замечание $2.2[11$, гл. 2]).

\section{3. Постановка нелокальных эллиптических задач трансмиссии}

3.1. Формула (2.1) порождает задачу, формально сопряженную к задаче (1.6), (1.7):

$$
\begin{gathered}
\Delta v_{j}(y)-v_{j}(y)=f_{j}(y), \quad y \in K_{j} \\
\left.v_{j}\right|_{\gamma_{j}}=g_{j}(y), \quad y \in \gamma_{j} \\
\left.v_{2}\right|_{\gamma}-\left.v_{1}\right|_{\gamma}=h_{1}(y),\left.\quad \frac{\partial v_{2}}{\partial n}\right|_{\gamma}-\left.\frac{\partial v_{1}}{\partial n}\right|_{\gamma}-\left.\sum_{k} \bar{a}_{k} \chi_{k}^{-1} \frac{\partial v_{k}}{\partial n_{k}}\left(\mathscr{G}_{k}^{-1} y\right)\right|_{\gamma}=h_{2}(y), \quad y \in \gamma .
\end{gathered}
$$


Задачу (3.1), (3.2) назовем нелокальной задачей трансмиссии в плоском угле $K$.

Положим

$$
\mathscr{E}_{-a+2}^{0}(K, \gamma)=E_{-a+2}^{0}(K) \times \prod_{j} E_{-a+2}^{3 / 2}\left(\gamma_{j}\right) \times \prod_{\nu} E_{-a+2}^{3 / 2-\nu}(\gamma)
$$

здесь и далее $\nu=0,1$. Обозначим также

$$
\mathscr{E}_{-a+2}^{2}(K)=\bigoplus_{j} E_{-a+2}^{2}\left(K_{j}\right)
$$

Рассмотрим ограниченньй оператор $\mathscr{M}_{K}: \mathscr{E}_{-a+2}^{2}(K) \rightarrow \mathscr{E}_{-a+2}^{0}(K, \gamma)$, действующий по формуле

$$
\mathscr{M}_{K} v=\left\{w-v,\left.v_{j}\right|_{\gamma_{j}},\left.v_{2}\right|_{\gamma}-\left.v_{1}\right|_{\gamma},\left.\frac{\partial v_{2}}{\partial n}\right|_{\gamma}-\left.\frac{\partial v_{1}}{\partial n}\right|_{\gamma}-\left.\sum_{k} \bar{a}_{k} \chi_{k}^{-1} \frac{\partial v_{k}}{\partial n_{k}}\left(\mathscr{G}_{k}^{-1} y\right)\right|_{\gamma}\right\} .
$$

Здесь и далее $v_{j}$ есть сужение функции $v \in \mathscr{E}_{-a+2}^{2}(K)$ на $K_{j}, w \equiv \Delta v_{j}$ при $y \in K_{j}$. (Отметим, что мы не можем считать $w \equiv \Delta v$ при $y \in K$, так как функция $v \in \mathscr{E}_{-a+2}^{2}(K)$ может иметь “разрыв" на $\gamma$. )

ЛЕмма 3.1. Для любих $g_{j} \in E_{-a+2}^{3 / 2}\left(\gamma_{j}\right) u h_{\nu} \in E_{-a+2}^{3 / 2-\nu}(\gamma)$ существует функиия $v \in \mathscr{E}_{-a+2}^{2}(K)$, удовлетворяюшая условиям (3.2) и такая, что

$$
\|v\|_{\mathscr{E}_{-a+2}^{2}(K)} \leqslant c\left(\sum_{j}\left\|g_{j}\right\|_{E_{-a+2}^{3 / 2}\left(\gamma_{j}\right)}+\sum_{\nu}\left\|h_{\nu}\right\|_{E_{-a+2}^{3 / 2-\nu}(\gamma)}\right)
$$

где с $>0$ не зависит от $g_{j} u h_{\nu}$.

ДокАЗАТЕЛЬСтво. В силу леммы $3.1^{\prime}[6]$ существуют $w_{j} \in E_{-a+2}^{2}\left(K_{j}\right)$ такие, что

$$
\begin{gathered}
\left.w_{j}\right|_{\gamma_{j}}=g_{j}(y), \quad y \in \gamma_{j} \\
\left\|w_{j}\right\|_{E_{-a+2}^{2}\left(K_{j}\right)} \leqslant k_{1}\left\|g_{j}\right\|_{E_{-a+2}^{3 / 2}\left(\gamma_{j}\right)^{2}}
\end{gathered}
$$

Повторяя доказательство леммы $3.1^{\prime}[6]$, построим $\widehat{w}_{2} \in E_{-a+2}^{2}\left(K_{2}\right)$ такую, что

$$
\begin{gathered}
\left.\widehat{w}_{2}\right|_{\gamma}=h_{1}(y),\left.\quad \frac{\partial \widehat{w}_{2}}{\partial n}\right|_{\gamma}=h_{2}(y)+\left.\sum_{k} \bar{a}_{k} \chi_{k}^{-1} \frac{\partial w_{k}}{\partial n_{k}}\left(\mathscr{G}_{k}^{-1} y\right)\right|_{\gamma}, \quad y \in \gamma, \\
\left\|\widehat{w}_{2}\right\|_{E_{-a+2}^{2}\left(K_{2}\right)} \leqslant k_{2}\left(\left\|h_{1}\right\|_{E_{-a+2}^{3 / 2}(\gamma)}+\left\|h_{2}+\left.\sum_{k} \bar{a}_{k} \chi_{k}^{-1} \frac{\partial v_{k}}{\partial n_{k}}\left(\mathscr{G}_{k}^{-1} y\right)\right|_{\gamma}\right\|_{E_{-a+2}^{1 / 2}(\gamma)}\right) .
\end{gathered}
$$

Введем функции $\zeta, \zeta_{j} \in \mathrm{C}_{0}^{\infty}(\mathbb{R}), \zeta_{j}(\varphi)=1$ для $\left|b_{j}-\varphi\right|<\varepsilon / 2, \zeta_{j}(\varphi)=1$ для $\left|b_{j}-\varphi\right|>\varepsilon ;$ $\zeta(\varphi)=1$ для $|b-\varphi|<\varepsilon / 2, \zeta(\varphi)=1$ для $|b-\varphi|>\varepsilon$. Здесь $\varepsilon=\min _{j}\left\{\left|b-b_{j}\right|\right\} / 4$. $\Phi$ ункции $\zeta, \zeta_{j}$ являются мультипликаторами в пространствах $E_{-a+2}^{2}\left(K_{j}\right)$. Отсюда и из (3.3)-(3.6) следует, что функция $v$ такая, что $v=v_{1}=\zeta_{1} w_{1}$ при $y \in K_{1}, v=v_{2}=$ $\zeta_{2} w_{2}+\zeta \widehat{w}_{2}$ при $y \in K_{2}$, удовлетворяет условиям леммы. 
3.2. Обозначим

$$
\mathscr{W}^{2}\left(b_{1}, b_{2}\right)=W^{2}\left(b_{1}, b\right) \oplus W^{2}\left(b, b_{2}\right) .
$$

Аналогично разделу 1 для задачи (3.1), (3.2) рассмотрим модельную оператор-функцию $\widetilde{\mathscr{M}}(\lambda): \mathscr{W}^{2}\left(b_{1}, b_{2}\right) \rightarrow \mathscr{W}^{0}\left[b_{1}, b_{2}\right]=L_{2}\left(b_{1}, b_{2}\right) \times \mathbb{C}^{2} \times \mathbb{C}^{2}$, определенную по формуле

$$
\begin{aligned}
\widetilde{\mathscr{M}}(\lambda) \widetilde{V}=\left\{\widetilde{W}(\varphi)-\lambda^{2} \widetilde{V}(\varphi),\left.\widetilde{V}_{j}(\varphi)\right|_{\varphi=b j},\left.\widetilde{V}_{2}(\varphi)\right|_{\varphi=b}-\left.\widetilde{V}_{1}(\varphi)\right|_{\varphi=b},\right. \\
\\
\left.\left.\quad \frac{d \widetilde{V}_{1}}{d \varphi}\right|_{\varphi=b}-\left.\frac{d \widetilde{V}_{2}}{d \varphi}\right|_{\varphi=b}-\left.\sum_{k}(-1)^{k} \bar{a}_{k} e^{-i \lambda \ln \chi_{k}} \frac{d \widetilde{V}_{k}}{d \varphi}\left(\varphi-\varphi_{k}\right)\right|_{\varphi=b}\right\} .
\end{aligned}
$$

Здесь $\widetilde{V}_{j}$ есть сужение функции $\widetilde{V} \in \mathscr{W}^{2}\left(b_{1}, b_{2}\right)$ на $K_{j}, \widetilde{W}(\varphi) \equiv\left(d^{2} / d \varphi^{2}\right) \widetilde{V}_{1}(\varphi)$ при $\varphi \in\left(b_{1}, b\right), \widetilde{W}(\varphi) \equiv\left(d^{2} / d \varphi^{2}\right) \widetilde{V}_{2}(\varphi)$ при $\varphi \in\left(b, b_{2}\right)$. Установим некоторые свойства оператор-функции $\widetilde{\mathscr{M}}(\lambda)$. Введем в гильбертовых пространствах $\mathscr{W}^{2}\left(b_{1}, b_{2}\right)$ и $\mathscr{W}^{0}\left[b_{1}, b_{2}\right]$ эквивалентные нормы, зависящие от параметра $\lambda \in \mathbb{C},|\lambda| \geqslant 1$ :

$$
\begin{aligned}
\|\widetilde{V}\|_{\mathscr{W}^{2}\left(b_{1}, b_{2}\right)} & =\left(\|\widetilde{V}\|_{\mathscr{W}^{2}\left(b_{1}, b_{2}\right)}^{2}+|\lambda|^{4}\|\widetilde{V}\|_{L_{2}\left(b_{1}, b_{2}\right)}^{2}\right)^{1 / 2} \\
\|\|\left\{\widetilde{F}, \widetilde{G}_{j}, \widetilde{H}_{\nu}\right\}\|\|_{\mathscr{W}^{0}\left[b_{1}, b_{2}\right]} & =\left(\|\widetilde{F}\|_{L_{2}\left(b_{1}, b_{2}\right)}^{2}+\sum_{j}|\lambda|^{3}\left|\widetilde{G}_{j}\right|^{2}+\sum_{\nu}|\lambda|^{3-2 \nu}\left|\widetilde{H}_{\nu}\right|^{2}\right)^{1 / 2} .
\end{aligned}
$$

Лемма 3.2. Для всех $\lambda \in \mathbb{C}$ оператор $\widetilde{\mathscr{M}}(\lambda)$ фредгольмов, ind $\widetilde{\mathscr{M}}(\lambda)=0 ;$ для любого $h \in \mathbb{R}$ существует $q_{0}>1$ такое, что для $\lambda \in J_{h, q_{0}}=\{\lambda \in \mathbb{C}: \operatorname{Im} \lambda=h$, $\left.|\operatorname{Re} \lambda| \geqslant q_{0}\right\}$ оператор $\widetilde{\mathscr{M}}(\lambda)$ имеет ограниченный обратный $\widetilde{\mathscr{M}}^{-1}(\lambda): \mathscr{W}^{0}\left[b_{1}, b_{2}\right] \rightarrow$ $\mathscr{W}^{2}\left(b_{1}, b_{2}\right) u$

$$
\left\|\widetilde{\mathscr{M}}^{-1}(\lambda) \widetilde{\Phi}\right\|_{\mathscr{W}^{2}\left(b_{1}, b_{2}\right)} \leqslant c\|\widetilde{\Phi}\|_{\mathscr{W}^{0}\left[b_{1}, b_{2}\right]}
$$

для всех $\widetilde{\Phi} \in \mathscr{W}^{0}\left[b_{1}, b_{2}\right]$, где с >0 не зависит от $\lambda$ и $\widetilde{\Phi} ;$ оператор-функиия $\widetilde{\mathscr{M}}^{-1}(\lambda)$ : $\mathscr{W}^{0}\left[b_{1}, b_{2}\right] \rightarrow \mathscr{W}^{2}\left(b_{1}, b_{2}\right)$ конечномероморфна.

ДокАЗАтЕЛЬство. В случае $a_{j}=0$, т.е. когда отсутствуют операторы, соответствующие нелокальным членам, обозначим $\widetilde{\mathscr{M}}(\lambda)$ через $\widetilde{\mathscr{M}}_{0}(\lambda)$. Следуя схеме, разработанной Аграновичем и Вишиком в работе [10], можно показать, что существуют такие $0<\varepsilon_{1}<\pi / 2$ и $q_{1}>1$, что при

$$
\lambda \in Q_{\varepsilon_{1}, q_{1}}=\left\{\lambda:|\lambda| \geqslant q_{1},|\arg \lambda| \leqslant \varepsilon_{1}\right\} \cup\left\{\lambda:|\lambda| \geqslant q_{1},|\arg \lambda-\pi| \leqslant \varepsilon_{1}\right\}
$$

существует обратньй оператор $\widetilde{\mathscr{M}}_{0}^{-1}(\lambda)$; при этом справедлива оценка

$$
\left\|\widetilde{\mathscr{M}}_{0}^{-1}(\lambda) \widetilde{\Phi}\right\|_{\mathscr{W}^{2}\left(b_{1}, b_{2}\right)} \leqslant k_{1}\|\widetilde{\Phi}\|_{\mathscr{W}^{0}\left[b_{1}, b_{2}\right]}
$$

для всех $\widetilde{\Phi} \in \mathscr{W}^{0}\left[b_{1}, b_{2}\right]$, где $k_{1}>0$ не зависит от $\lambda$ и $\widetilde{\Phi}$.

Введем оператор $\widetilde{\mathscr{M}}_{t}(\lambda)=\widetilde{\mathscr{M}}_{0}(\lambda)+t\left(\widetilde{\mathscr{M}}(\lambda)-\widetilde{\mathscr{M}}_{0}(\lambda)\right), 0 \leqslant t \leqslant 1$. Докажем, что для любого $h \in \mathbb{R}$ существует такое $q_{0}>0$, что при $\lambda \in J_{h, q_{0}}$ и $0 \leqslant t \leqslant 1$

$$
k_{2}\left\|\widetilde { \mathscr { M } } _ { t } ( \lambda ) \widetilde { V } \left|\left\|_{\mathscr{W}^{0}\left[b_{1}, b_{2}\right]} \leqslant\right\| \tilde{V}\left\|_{\mathscr{W}^{2}\left(b_{1}, b_{2}\right)} \leqslant k_{3} \mid\right\| \widetilde{\mathscr{M}}_{t}(\lambda) \widetilde{V} \|_{\mathscr{W}^{0}\left[b_{1}, b_{2}\right]}\right.\right.
$$


для всех $\widetilde{V} \in \mathscr{W}^{2}\left(b_{1}, b_{2}\right)$, где $k_{2}, k_{3}>0$ не зависят от $\lambda, t$ и $V$.

Обозначим $\widetilde{\mathscr{M}}_{t}(\lambda) \widetilde{V}=\widetilde{\Phi}$. Тогда $\widetilde{\mathscr{M}}_{0}(\lambda) \widetilde{V}=\widetilde{\Phi}+\widetilde{\Psi}$, где

$$
\widetilde{\Psi}=\left(0,0,0,0,\left.t \sum_{k}(-1)^{k} \bar{a}_{k} e^{-i \lambda \ln \chi_{k}} \frac{d \widetilde{V}_{k}}{d \varphi}\left(\varphi-\varphi_{k}\right)\right|_{\varphi=b}\right) .
$$

В силу (3.8)

$$
\|\widetilde{V}\|_{\mathscr{W}^{2}\left(b_{1}, b_{2}\right)} \leqslant k_{1}\|\widetilde{\Phi}+\widetilde{\Psi}\|_{\mathscr{W}^{0}\left[b_{1}, b_{2}\right]} .
$$

Положим $\varepsilon=\min _{j}\left\{\left|b-b_{j}\right|\right\} / 4$ и выберем $q_{0} \geqslant q_{1}$ так, чтобы $J_{h, q_{0}} \subset Q_{\varepsilon_{1}, q_{1}}$. Тогда, используя неравенства (1.3), (1.4), получим

$$
\begin{aligned}
I_{1} & =|\lambda|^{1 / 2}\left|\bar{a}_{1} e^{-i \lambda \ln \chi_{1}} \frac{d \widetilde{V}_{1}}{d \varphi}\left(\varphi-\varphi_{1}\right)\right|_{\varphi=b} \mid \\
& \leqslant k_{4}\left\{\left\|\frac{d \widetilde{V}_{1}}{d \varphi}\right\|_{W^{1}\left(b_{1}, b_{1}+\varepsilon / 2\right)}+|\lambda|\left\|\frac{d \widetilde{V}_{1}}{d \varphi}\right\|_{L^{2}\left(b_{1}, b_{1}+\varepsilon / 2\right)}\right\} \leqslant k_{5} \mid\left\|\widetilde{V}_{1}\right\|_{W^{2}\left(b_{1}, b_{1}+\varepsilon / 2\right)} .
\end{aligned}
$$

Пусть $q_{1}$ настолько велико, что в области $Q_{\varepsilon_{1}, q_{1}}$ имеет место теорема 4.1 [10, гл. 1 ]. Тогда из неравенства (3.11) и теоремы 4.1 [10, гл. 1] при помоши формулы Лейбница и интерполяционного неравенства (1.3) получим

$$
\begin{aligned}
I_{1} & \leqslant k_{5}\left|\left\|\zeta_{1} \widetilde{V}_{1} \mid\right\|_{W^{2}\left(b_{1}, b_{1}+\varepsilon / 2\right)} \leqslant k_{6}\left(\|||\left(\frac{d^{2}}{d \varphi^{2}}-\lambda^{2}\right)\left(\zeta_{1} \widetilde{V}_{1}\right)||_{L_{2}\left(b_{1}, b\right)}+|\lambda|^{3 / 2}\left|\widetilde{V}_{1}(\varphi)\right|_{\varphi=b_{1}}\right)\right. \\
& \leqslant k_{7}\left(\left.||\left|\left(\frac{d^{2}}{d \varphi^{2}}-\lambda^{2}\right) \widetilde{V}_{1}\right|\right|_{L_{2}\left(b_{1}, b\right)}+|\lambda|^{-1}|| \widetilde{V}_{1}||_{W^{2}\left(b_{1}, b\right)}+|\lambda|^{3 / 2}\left|\widetilde{V}_{1}(\varphi)\right|_{\varphi=b_{1}}\right),
\end{aligned}
$$

где $\zeta_{1}$ та же, что и в доказательстве леммы 3.1. Аналогично (3.11), (3.12) оценивается $I_{2}=|\lambda|^{1 / 2}\left|\bar{a}_{2} e^{-i \lambda \ln \chi_{2}}\left(d \widetilde{V}_{2} / d \varphi\right)\left(\varphi-\varphi_{2}\right)\right|_{\varphi=b} \mid:$

$$
I_{2} \leqslant k_{8}\left(\left\|\left.||\left(\frac{d^{2}}{d \varphi^{2}}-\lambda^{2}\right) \widetilde{V}_{2}||\right|_{L_{2}\left(b, b_{2}\right)}+\left.|\lambda|^{-1}|| \widetilde{V}_{2}\left|\|_{W^{2}\left(b, b_{2}\right)}+\right| \lambda\right|^{3 / 2}\left|\widetilde{V}_{2}(\varphi)\right|_{\varphi=b_{2}}\right) .\right.
$$

Считая, что $q_{0}$ достаточно велико, из (3.10), (3.12), (3.13) получим правую часть неравенства (3.9). Левая часть неравенства (3.9) очевидна. Применяя стандартньй метод продолжения по параметру $t$ (см. доказательство теоремы $7.1[12$, гл. $2, \S 7]$ ), в силу неравенства (3.9) и существования обратного оператора $\widetilde{\mathscr{M}}_{0}^{-1}(\lambda)$ при $\lambda \in Q_{\varepsilon_{1}}, q_{1}$ убеждаемся в том, что для $\lambda \in J_{h, q_{0}}$ оператор $\widetilde{\mathscr{M}}(\lambda)$ также имеет ограниченный обратный и при этом вьполняется неравенство (3.7). Используя теорему 16.4 [13] об устойчивости индекса фредгольмова оператора по отношению к компактным возмущениям, нетрудно показать, что оператор $\widetilde{\mathscr{M}}(\lambda)$ фредгольмов для всех $\lambda \in \mathbb{C}$ и ind $\widetilde{\mathscr{M}}(\lambda)=0$. Отсюда, из существования $\widetilde{\mathscr{M}}^{-1}(\lambda)$ при $\lambda \in J_{h, q_{0}}$ и теоремы 1 [14] заключаем, что оператор-функция $\widetilde{\mathscr{M}}^{-1}(\lambda)$ конечномероморфна.

Аналогично лемме 2.2 [3] при помощи (3.10)-(3.13) доказьвается 
Лемма 3.3. Для любого $0<\varepsilon<1 / \max \left|\ln \chi_{j}\right|$ существует $q>1$ такое, что множество $\{\lambda \in \mathbb{C}:|\operatorname{Im} \lambda| \leqslant \varepsilon \ln |\operatorname{Re} \lambda|,|\operatorname{Re} \lambda| \geqslant q\}$ не содержит полюсов оператор-функиии $\widetilde{\mathscr{M}}^{-1}(\lambda) ;$ для каждого полюса $\lambda_{0}$ оператор-функиии $\widetilde{\mathscr{M}}^{-1}(\lambda)$ существует $\delta>0$ такое, ито множество $\left\{\lambda \in \mathbb{C}: 0<\left|\operatorname{Im} \lambda-\operatorname{Im} \lambda_{0}\right|<\delta\right\}$ не содержит полюсов оператор-функиии $\widetilde{\mathscr{M}}^{-1}(\lambda)$.

3.3. Рассмотрим для функций $v_{j}$ вспомогательную систему двух уравнений

$$
\Delta v_{j}(y)=\hat{f}_{j}(y), \quad y \in K_{j}
$$

с краевыми условиями и нелокальными условиями трансмиссии (3.2). Введем пространство

$$
\mathscr{H}_{-a+2}^{0}(K, \gamma)=H_{-a+2}^{0}(K) \times \prod_{j} H_{-a+2}^{3 / 2}\left(\gamma_{j}\right) \times \prod_{\nu} H_{-a+2}^{3 / 2-\nu}(\gamma)
$$

Обозначим также $\mathscr{H}_{-a+2}^{2}(K)=\bigoplus_{j} H_{-a+2}^{2}\left(K_{j}\right)$.

Аналогично теореме 2.1 [3] из леммы 3.2 получим следующий результат.

Лемма 3.4. Пусть прямая $\operatorname{Im} \lambda=-a+1$ не содержит полюсов оператор-функции $\widetilde{\mathscr{M}}^{-1}(\lambda)$. Тогда нелокальная задача трансмиссии (3.14), (3.2) имеет единственное решение $v \in \mathscr{H}_{-a+2}^{2}(K)$ для любой правой части $\left\{\hat{f}, g_{j}, h_{\nu}\right\} \in \mathscr{H}_{-a+2}^{0}(K, \gamma)$ и выполнена оценка

$$
\|v\|_{\mathscr{H}_{-a+2}^{2}(K)} \leqslant c\left\|\left\{\hat{f}, g_{j}, h_{\nu}\right\}\right\|_{\mathscr{H}_{-a+2}^{0}(K, \gamma)},
$$

где с $>0$ не зависит от $\left\{\hat{f}, g_{j}, h_{\nu}\right\} ; v(y) \equiv v_{j}(y), \hat{f}(y) \equiv \hat{f}_{j}(y)$ nри $y \in K_{j}$.

Лемма 3.4 понадобится в разделе 4 при выводе априорных оценок решений задачи (3.1), (3.2).

\section{4. Априорные оценки решений нелокальных эллиптических задач}

4.1. Введем множество

$$
\Gamma=\left\{x=(y, z): r>0, \varphi=b, z \in \mathbb{R}^{n-2}\right\} .
$$

Множество Г является носителем нелокальных данных в задаче $(1.1),(1.2)$. Обозначим

$$
\begin{aligned}
& \Omega_{1}=\left\{x=(y, z): r>0, b_{1}<\varphi<b, z \in \mathbb{R}^{n-2}\right\}, \\
& \Omega_{2}=\left\{x=(y, z): r>0, b<\varphi<b_{2}, z \in \mathbb{R}^{n-2}\right\} .
\end{aligned}
$$

Пусть $n_{j}$ есть нормаль к $\Gamma_{j}$, направленная вне области $\Omega_{j}, n$ - нормаль к $\Gamma$, направленная вне области $\Omega_{2}$. Положим $d_{1}=\min \left\{1, \chi_{j}^{-1}\right\} / 2, d_{2}=2 \max \left\{1, \chi_{j}^{-1}\right\}$;

$$
\begin{aligned}
& \Omega_{j}^{p}=\Omega_{j} \cap\left\{x=(y, z): r_{1} d_{1}^{3-p}<r<r_{2} d_{2}^{3-p},|z|<2^{-p-1}\right\}, \\
& \Omega^{p}=\Omega \cap\left\{x=(y, z): r_{1} d_{1}^{3-p}<r<r_{2} d_{2}^{3-p},|z|<2^{-p-1}\right\},
\end{aligned}
$$

где $p=0, \ldots, 3 ; 0<r_{1}<r_{2}$.

Обозначим

$$
\mathscr{W}^{2}\left(\Omega^{p}\right)=\bigoplus_{j} W^{2}\left(\Omega_{j}^{p}\right) .
$$

Пусть $V_{j}$ есть сужение $V \in \mathscr{W}^{2}\left(\Omega^{p}\right)$ на $\Omega_{j}^{p}$. 
Лемма 4.1. Для всех $V \in \mathscr{W}^{2}\left(\Omega^{0}\right) u|\lambda| \geqslant 1$ выполнено неравенство

$$
\begin{aligned}
\|V\|_{\mathscr{W}^{2}\left(\Omega^{3}\right)} \leqslant & c\left(\sum_{j}\left\|\Delta V_{j}\right\|_{W^{2}\left(\Omega_{j}^{0}\right)}+\sum_{j}\left\|\left.V_{j}\right|_{\Gamma_{j}}\right\|_{W^{3 / 2}\left(\Gamma_{j} \cap \bar{\Omega}^{0}\right)}\right. \\
& +\left\|\left.V_{2}\right|_{\Gamma}-\left.V_{1}\right|_{\Gamma}\right\|_{W^{3 / 2}\left(\Gamma \cap \bar{\Omega}^{0}\right)} \\
& +\left\|\left.\frac{\partial V_{2}}{\partial n}\right|_{\Gamma}-\left.\frac{\partial V_{1}}{\partial n}\right|_{\Gamma}-\left.\sum_{k} \bar{a}_{k} \chi_{k}^{-1} \frac{\partial V_{k}}{\partial n_{k}}\left(\mathscr{G}_{k}^{-1} y, z\right)\right|_{\Gamma}\right\|_{W^{1 / 2}\left(\Gamma \cap \bar{\Omega}^{0}\right)} \\
& \left.+|\lambda|^{-1}\|V\|_{\mathscr{W}^{2}\left(\Omega^{0}\right)}+|\lambda|\|V\|_{L_{2}\left(\Omega^{0}\right)}\right)
\end{aligned}
$$

где $c>0$ не зависит от $\lambda u V$.

ДокАЗАТЕЛЬСтво. Из теоремы 1 [15] следует априорная оценка

$$
\begin{aligned}
& \|V\|_{W^{2}\left(\Omega^{3}\right)} \leqslant k_{1}\left(\sum_{j}\left\|\Delta V_{j}\right\|_{W^{2}\left(\Omega_{j}^{2}\right)}+\sum_{j}\left\|\left.V_{j}\right|_{\Gamma_{j}}\right\|_{W^{3 / 2}\left(\Gamma_{j} \cap \bar{\Omega}^{2}\right)}\right. \\
& \left.\quad+\left\|\left.V_{2}\right|_{\Gamma}-\left.V_{1}\right|_{\Gamma}\right\|_{W^{3 / 2}\left(\Gamma \cap \bar{\Omega}^{2}\right)}+\left\|\left.\frac{\partial V_{2}}{\partial n}\right|_{\Gamma}-\left.\frac{\partial V_{1}}{\partial n}\right|_{\Gamma}\right\|_{W^{1 / 2}\left(\Gamma \cap \bar{\Omega}^{2}\right)}+\|V\|_{L_{2}\left(\Omega^{2}\right)}\right) .
\end{aligned}
$$

Обозначим

$$
W=\sum_{k} \bar{a}_{k} \chi_{k}^{-1} \frac{\partial\left(\zeta_{k} V_{k}\right)}{\partial n_{k}}\left(\mathscr{G}_{k}^{-1} y, z\right)
$$

где $\zeta_{k}$ те же, что и в доказательстве леммы 3.1. Очевидно,

$$
\left.W\right|_{\Gamma \cap \bar{\Omega}^{2}}=\left.\sum_{k} \bar{a}_{k} \chi_{k}^{-1} \frac{\partial V_{k}}{\partial n_{k}}\left(\mathscr{G}_{k}^{-1} y, z\right)\right|_{\Gamma \cap \bar{\Omega}^{2}}
$$

Применяя теорему 5.1 [11, гл. 2], формулу Лейбнища и неравенство (1.3), получим

$$
\begin{aligned}
& \left\|\left.W\right|_{\Gamma \cap \bar{\Omega}^{2}}\right\|_{W^{1 / 2}\left(\Gamma \cap \bar{\Omega}_{j}^{2}\right)} \leqslant k_{2} \sum_{k}\left\|\zeta_{k} V_{k}\right\|_{W^{2}\left(\Omega_{k}^{1}\right)} \\
& \quad \leqslant k_{3} \sum_{k}\left\{\left\|\Delta V_{k}\right\|_{L_{2}\left(\Omega_{k}^{0}\right)}+\left\|\left.V_{k}\right|_{\Gamma_{k}}\right\|_{W^{3 / 2}\left(\Gamma_{k} \cap \bar{\Omega}_{k}^{0}\right)}+|\lambda|^{-1}\left\|V_{k}\right\|_{W^{2}\left(\Omega_{k}^{0}\right)}+|\lambda|\left\|V_{k}\right\|_{L_{2}\left(\Omega_{k}^{0}\right)}\right\} .
\end{aligned}
$$

Из (4.2)-(4.4) следует неравенство (4.1).

Лемма 4.2. Пусть $v_{j} \in W_{\mathrm{loc}}^{2}\left(\bar{K}_{j} \backslash\{0\}\right)$ - решение нелокальной задачи трансмиссии (3.14), (3.2) такое, что $v \in H_{-a}^{0}(K), a\left\{\hat{f}, g_{j}, h_{\nu}\right\} \in \mathscr{H}_{-a+2}^{0}(K, \gamma)$. Тогда $v \in \mathscr{H}_{-a+2}^{2}(K)$, при этом

$$
\|v\|_{\mathscr{H}_{-a+2}^{2}(K)} \leqslant c\left(\left\|\left\{\hat{f}, g_{j}, h_{\nu}\right\}\right\|_{\mathscr{H}_{-a+2}^{0}(K, \gamma)}+\|v\|_{H_{-a}^{0}(K)}\right)
$$

әде $c>0$ не зависит от $v$. 
ДокАЗАТЕЛЬСтво. Аналогично доказательству леммы 3.2 [3] доказательство леммы 4.2 следует из леммы 3.1 и аналога леммы 4.1 при $n=2$.

4.2. Пусть

$$
\begin{aligned}
& K_{j}^{p s}=K_{j} \cap\left\{r_{1} d_{1}^{3-p} \cdot 2^{s}<r<r_{2} d_{2}^{3-p} \cdot 2^{s}\right\}, \\
& K^{p s}=K \cap\left\{r_{1} d_{1}^{3-p} \cdot 2^{s}<r<r_{2} d_{2}^{3-p} \cdot 2^{s}\right\},
\end{aligned}
$$

где $0<r_{1}<r_{2} ; s \geqslant 1 ; p=0, \ldots, 3$.

Обозначим

$$
\mathscr{W}^{2}\left(K^{p s}\right)=\bigoplus_{j} W^{2}\left(K_{j}^{p s}\right)
$$

Пусть $v_{j}$ есть сужение $v \in \mathscr{W}^{2}\left(K^{p s}\right)$ на $K_{j}^{p s}$.

Лемма 4.3. Пусть $s \geqslant 1$. Предположим, что $v \in \mathscr{W}^{2}\left(K^{0 s}\right)$,

$$
\begin{gathered}
\left.v_{j}\right|_{\gamma_{j}}=0, \quad y \in \gamma_{j} \cap \bar{K}_{j}^{0 s},\left.\quad v_{2}\right|_{\gamma}-\left.v_{1}\right|_{\gamma}=0, \quad y \in \gamma \cap K^{0 s}, \\
\left.\frac{\partial v_{2}}{\partial n}\right|_{\gamma}-\left.\frac{\partial v_{1}}{\partial n}\right|_{\gamma}-\left.\sum_{k} \bar{a}_{k} \chi_{k}^{-1} \frac{\partial v_{k}}{\partial n_{k}}\left(\mathscr{G}_{k}^{-1} y\right)\right|_{\gamma}=0, \quad y \in \gamma \cap K^{0 s} .
\end{gathered}
$$

Тогда $\partial л я|\lambda| \geqslant 1$

$$
\begin{aligned}
2^{s(-a+2)}\|v\|_{\mathscr{W}^{2}\left(K^{3 s}\right)} \leqslant & c\left(2^{s(-a+2)} \sum_{j}\left\|\Delta v_{j}-v_{j}\right\|_{L_{2}\left(K_{j}^{0 s}\right)}+|\lambda|^{-1} 2^{s(-a+2)}\|v\|_{\mathscr{W}^{2}\left(K^{0 s}\right)}\right. \\
& \left.+|\lambda| 2^{s(-a)}\|v\|_{L_{2}\left(K^{0 s}\right)}\right)
\end{aligned}
$$

где с >0 не зависит от $v, \lambda u s$.

ДокаЗАТЕльСтво. Аналогично доказательству леммы 3.3 [3] лемма 4.3 доказывается путем подстановки функции $V(y, z)=e^{i \cdot 2^{s}(\theta, z)} v(y), \theta \in \mathbb{R}^{n-2},|\theta|=1$, в неравенство (4.1), последующей заменены переменных $y^{\prime}=2^{s} y$ и умножения обеих частей полученного неравенства на $2^{s(-a)}$.

Tеорема 4.1. Пусть $v_{j} \in W_{\text {loc }}^{2}\left(\bar{K}_{j} \backslash\{0\}\right)$ - решение задачи (3.1), (3.2) такое, что $v \in E_{-a}^{0}(K), a\left\{f, g_{j}, h_{\nu}\right\} \in \mathscr{E}_{-a+2}^{0}(K, \gamma)$. Тогдa $v \in \mathscr{E}_{-a+2}^{2}(K)$, при этом

$$
\|v\|_{\mathscr{E}_{-a+2}^{2}(K)} \leqslant c\left(\left\|\left\{f, g_{j}, h_{\nu}\right\}\right\|_{\mathscr{E}_{-a+2}^{0}(K, \gamma)}+\|v\|_{E_{-a}^{0}(K)}\right)
$$

где с $>0$ не зависит от $v ; v(y) \equiv v_{j}(y), f(y) \equiv f_{j}(y)$ при $y \in K_{j}$. 
ДокАЗАТЕЛЬСТво. 1) Согласно лемме 3.1 достаточно рассмотреть случай $\left\{g_{j}, h_{\nu}\right\}$ $=0$. Пусть $r_{1}=d_{1}, r_{2}=d_{2}$. Тогда

$$
\begin{aligned}
& K_{j}^{p s}=K_{j} \cap\left\{d_{1}^{4-p} \cdot 2^{s}<r<d_{2}^{4-p} \cdot 2^{s}\right\}, \\
& K^{p s}=K \cap\left\{d_{1}^{4-p} \cdot 2^{s}<r<d_{2}^{4-p} \cdot 2^{s}\right\},
\end{aligned}
$$

где $s \geqslant 1 ; p=0, \ldots, 3$. Обозначим также $K_{j}^{30}=K_{j} \cap\left\{r<d_{2}\right\}$. Введем функции $\psi$, $\hat{\psi} \in C^{\infty}(\mathbb{R}), \psi(r)=1$ при $r<d_{2}, \psi(r)=0$ при $r>2 d_{2} ; \hat{\psi}(r)=1$ при $r<2 d_{2}^{2}, \hat{\psi}(r)=0$ при $r>3 d_{2}^{2}$.

По лемме 4.2

$$
\|v\|_{\mathscr{E}_{-a+2}^{2}\left(K_{j}^{30}\right)} \leqslant k_{1}\|\psi v\|_{\mathscr{H}_{-a+2}^{2}(K)} \leqslant k_{2}\left(\sum_{j}\left\|\Delta\left(\psi v_{j}\right)\right\|_{H_{-a+2}^{0}\left(K_{j}\right)}+\|\psi v\|_{H_{-a}^{0}(K)}\right) .
$$

Оценим $\left\|\Delta\left(\psi v_{j}\right)\right\|_{H_{-a+2}^{0}\left(K_{j}\right)}$. Используя формулу Лейбница и ограничение на носители функций $\psi, \hat{\psi}$, получим

$$
\begin{aligned}
\left\|\Delta\left(\psi v_{j}\right)\right\|_{H_{-a+2}^{0}\left(K_{j}\right)} & \leqslant\left\|\Delta\left(\psi v_{j}\right)-\psi v_{j}\right\|_{H_{-a+2}^{0}\left(K_{j}\right)}+\left\|\psi v_{j}\right\|_{H_{-a+2}^{0}\left(K_{j}\right)} \\
& \leqslant k_{3}\left(\left\|\Delta v_{j}-v_{j}\right\|_{E_{-a+2}^{0}\left(K_{j}\right)}+\left\|\hat{\psi} v_{j}\right\|_{H_{-a+1}^{1}\left(K_{j}\right)}\right) .
\end{aligned}
$$

Неравенства (4.8), (4.9) и интерполящионное неравенство (1.5) дают

$$
\|v\|_{\mathscr{E}_{-a+2}^{2}\left(K^{30}\right)} \leqslant k_{4}\left(\sum_{j}\left\|f_{j}\right\|_{E_{-a+2}^{0}\left(K_{j}\right)}+|\lambda|^{-1}\|v\|_{\mathscr{E}_{-a+2}^{2}(K)}+|\lambda|\|v\|_{E_{-a}^{0}(K)}\right) .
$$

2) В силу леммы 4.3 при $s \geqslant 1$

$$
\|v\|_{\mathscr{E}_{-a+2}^{2}\left(K^{3 s}\right)} \leqslant k_{5}\left(\sum_{j}\left\|f_{j}\right\|_{E_{-a+2}^{0}\left(K_{j}^{0 s}\right)}+|\lambda|^{-1}\|v\|_{\mathscr{E}_{-a+2}^{2}\left(K^{0 s}\right)}+|\lambda|\|v\|_{E_{-a}^{0}\left(K^{0 s}\right)}\right) .
$$

Складьвая (4.10) и (4.11) для $s \geqslant 1$, при достаточно больших $|\lambda|$ получим (4.7).

Аналогично теореме 3.1 [3.1] из лемм $3.1,3.4$ и 4.3 вьводится следующий результат.

ТЕОРема 4.2. Пусть на прямой $\operatorname{Im} \lambda=-a+1$ нет полюсов оператор-функиии $\widetilde{\mathscr{M}}^{-1}(\lambda)$. Тогда для $v \in \mathscr{E}_{-a+2}^{2}(K)$ выполнена оценка

$$
\|v\|_{\mathscr{E}_{-a+2}^{2}(K)} \leqslant c\left(\left\|\mathscr{M}_{K} v\right\|_{\mathscr{E}_{-a+2}^{0}(K, \gamma)}+\|v\|_{L_{2}\left(K \cap S^{\prime}\right)}\right)
$$

где $S^{\prime}=\left\{y \in \mathbb{R}^{2}: 0<R_{1}^{\prime}<r<R_{2}^{\prime}\right\} ;$ c $>0$ не зависит от $v$.

Обратно, если для всех $v \in \mathscr{E}_{-a+2}^{2}(K)$ имеет место оченка (4.12), то на прямой $\operatorname{Im} \lambda=-a+1$ нет полюсов оператор-функиии $\widetilde{\mathscr{M}}^{-1}(\lambda)$.

Из теоремы 4.2 следует, что $\mathscr{M}_{K}$ имеет конечномерное ядро и замкнутьй образ. Отметим, что это утверждение не следует из оценки (4.7) (справедливой, даже если на прямой $\operatorname{Im} \lambda=-a+1$ есть полюсы $\left.\widetilde{\mathscr{M}}^{-1}(\lambda)\right)$, так как вложение $\mathscr{E}_{-a+2}^{2}(K) \subset E_{-a}^{0}(K)$ непрерьвно, но не компактно.

Далее будет установлена связь между ядрами операторов $\mathscr{M}_{K}$ и $\mathscr{L}_{K}^{*}\left(\mathscr{L}_{K}^{*}\right.$ - оператор, сопряженньй с $\mathscr{L}_{K}$ ). Для изучения оператора $\mathscr{L}_{K}^{*}$ нам понадобится утверждение об априорных оценках и гладкости решений одной вспомогательной задачи. Сформулируем это утверждение в следующем пункте. 
4.3. Рассмотрим ограниченньй оператор

$$
\mathscr{L}: W^{2}\left(\mathbb{R}^{2}\right) \rightarrow L_{2}\left(\mathbb{R}^{2}\right) \times \prod_{j} W^{3 / 2}\left(\mathbb{R}^{1}\right)
$$

действующий по формуле

$$
\mathscr{L} U=\left(\Delta U,\left.U\right|_{x_{2}=0},\left.U\right|_{x_{2}=0}\right) .
$$

Отметим, что задача, соответствующая оператору $\mathscr{L}$, носит искусственный характер: это не краевая задача (так как решение $U$ ищется во всем $\mathbb{R}^{2}$ ) и не задача трансмиссии (так как на прямой $\left\{x_{2}=0\right\}$ задаются не условия сопряжения, а след функции $U$, да к тому же дважды). Однако при вьводе априорных оценок для решений сопряженных нелокальных задач - в следующем пункте - мы столкнемся с задачей именно такого типа, что объясняется спецификой используемого метода "отделения нелокальностей".

Введем ограниченньй оператор

$$
\mathscr{L}^{*}: L_{2}\left(\mathbb{R}^{2}\right) \times \prod_{j} W^{-3 / 2}\left(\mathbb{R}^{1}\right) \rightarrow W^{-2}\left(\mathbb{R}^{2}\right),
$$

сопряженньй к $\mathscr{L}$. Оператор $\mathscr{L}^{*}$ действует на $\left\{f, g_{j}\right\} \in L_{2}\left(\mathbb{R}^{2}\right) \times \prod_{j} W^{-3 / 2}\left(\mathbb{R}^{1}\right)$ по формуле

$$
\left\langle U, \mathscr{L}^{*}\left\{f, g_{j}\right\}\right\rangle=(\Delta U, f)_{\mathbb{R}^{2}}+\sum_{j}\left\langle\left. U\right|_{x_{2}=0}, g_{j}\right\rangle_{\mathbb{R}^{1}} \quad \text { для любых } U \in W^{2}\left(\mathbb{R}^{2}\right),
$$

где $(\cdot, \cdot)_{\mathbb{R}^{2}}$ обозначает скалярное произведение в $L_{2}\left(\mathbb{R}^{2}\right),\langle\cdot, \cdot\rangle_{\mathbb{R}^{1}}$ обозначает полуторалинейную форму на паре пространств $W^{3 / 2}\left(\mathbb{R}^{1}\right), W^{-3 / 2}\left(\mathbb{R}^{1}\right)$.

Обозначим $\mathbb{R}_{+}^{2}=\left\{x \in \mathbb{R}^{2}: x_{2}>0\right\}, \mathbb{R}_{-}^{2}=\left\{x \in \mathbb{R}^{2}: x_{2}<0\right\}$. Для целых $l \geqslant 0$ введем пространство

$$
\mathscr{W}^{l}\left(\mathbb{R}^{2}\right)=W^{l}\left(\mathbb{R}_{+}^{2}\right) \oplus W^{l}\left(\mathbb{R}_{-}^{2}\right) .
$$

ЛЕммА 4.4. При всяком иелом фиксированном $l \geqslant 0$ если

$$
\left\{f, g_{j}\right\} \in L_{2}\left(\mathbb{R}^{2}\right) \times \prod_{j} W^{-3 / 2+l}\left(\mathbb{R}^{1}\right), \quad \mathscr{L}^{*}\left\{f, g_{j}\right\} \in\left\{\begin{array}{lll}
W^{-2+l}\left(\mathbb{R}^{2}\right) & n p u & l<2 \\
\mathscr{W}^{-2+l}\left(\mathbb{R}^{2}\right) & n p u & l \geqslant 2
\end{array}\right.
$$

$m o f \in \mathscr{W}^{l}\left(\mathbb{R}^{2}\right) u$

$$
\|f\|_{\mathscr{W}^{l}\left(\mathbb{R}^{2}\right)} \leqslant c_{l}\left(\left\|\mathscr{L}^{*}\left\{f, g_{j}\right\}\right\|_{-2+l}+\|f\|_{W^{-1}\left(\mathbb{R}^{2}\right)}+\sum_{j}\left\|g_{j}\right\|_{W^{-3 / 2+l}\left(\mathbb{R}^{1}\right)}\right)
$$

$2 \partial e$

$$
\|\cdot\|_{-2+l}=\left\{\begin{array}{lll}
\|\cdot\|_{W^{-2+l}\left(\mathbb{R}^{2}\right)} & n p u & l<2, \\
\|\cdot\|_{\mathscr{W}-2+l}\left(\mathbb{R}^{2}\right) & n p u & l \geqslant 2,
\end{array}\right.
$$

$c_{l}>0$ не зависят от $\left\{f, g_{j}\right\}$. 
ДокАЗАТЕЛЬСтво проводится по схеме [11, гл. 2] (см. теоремы 4.1, 4.3 [11, гл. 2]).

Отметим, что в отличие от модельньх задач во всем пространстве (см. [11, гл. $2, \S 3]$ в данном случае оператор $\mathscr{L}^{*}$ содержит распределения с носителями на прямой $\left\{x_{2}=0\right\}$. В связи с этим гладкость функции $f$ может нарушаться на прямой $\left\{x_{2}=0\right\}$, даже если $\mathscr{L}^{*}\left\{f, g_{j}\right\}$ бесконечно гладкая в $\mathbb{R}^{2}$. Более того, лемма 4.4 означает, что для повьшения гладкости функции $f$ в $\mathbb{R}_{+}^{2}$ и $\mathbb{R}_{-}^{2}$ необходимо потребовать дополнительную гладкость не только от $\mathscr{L}^{*}\left\{f, g_{j}\right\}$, но и от распределений $g_{j}$.

4.4. Теперь изучим оператор, сопряженньй к $\mathscr{L}_{K}$. Для целых $l \geqslant 0$ обозначим через $\left(E_{a}^{l}(K)\right)^{*},\left(E_{a}^{l+1 / 2}\left(\gamma_{j}\right)\right)^{*}$ и $\left(E_{a}^{l+1 / 2}(\gamma)\right)^{*}$ пространства, сопряженные с $E_{a}^{l}(K)$, $E_{a}^{l+1 / 2}\left(\gamma_{j}\right)$ и $E_{a}^{l+1 / 2}(\gamma)$ относительно скалярных произведений в $L_{2}(K), L_{2}\left(\gamma_{j}\right)$ и $L_{2}(\gamma)$ соответственно. Очевидно, $\left(E_{a}^{0}(K)\right)^{*}=E_{-a}^{0}(K)$.

Положим $\hat{\gamma}_{j}=\left\{y: \varphi=b_{j}\right.$ или $\left.\varphi=b_{j}+\pi\right\}, \hat{\gamma}=\{y: \varphi=b$ или $\varphi=b+\pi\}$. Очевидно, $\gamma_{j} \subset \hat{\gamma}_{j}, \gamma \subset \hat{\gamma}$. Для целых $l \geqslant 0$ обозначим через $W_{\bar{K}}^{-l}\left(\mathbb{R}^{2}\right), W^{-l-1 / 2}\left(\hat{\gamma}_{j}\right)$ и $W^{-l-1 / 2}(\hat{\gamma})$ пространства, сопряженные с $W^{l}(K), W^{l+1 / 2}\left(\hat{\gamma}_{j}\right)$ и $W^{l+1 / 2}(\hat{\gamma})$ соответственно.

Введем функции $\psi_{p} \in C_{0}^{\infty}\left(\mathbb{R}^{2}\right)$ такие, что $\psi_{p}(y)=1$ при $r_{1} d_{1}^{3-p}<r<r_{2} d_{2}^{3-p}$, $\psi_{p}(y)=0$ при $r<2 r_{1} d_{1}^{3-p} / 3$ и $r>3 r_{2} d_{2}^{3-p} / 2$. Здесь $0<r_{1}<r_{2} ; p=0, \ldots, 3$.

Для $g_{j} \in\left(E_{a}^{l+1 / 2}\left(\gamma_{j}\right)\right)^{*}$ обозначим через $\psi_{p} g_{j}$ распределение из $W^{-l-1 / 2}\left(\hat{\gamma}_{j}\right)$, определяемое соотношением $\left\langle u_{\hat{\gamma}_{j}}, \psi_{p} g_{j}\right\rangle_{\hat{\gamma}_{j}}=\left\langle\psi_{p} u_{\hat{\gamma}_{j}}, g_{j}\right\rangle_{\gamma_{j}}$ для всех $u_{\hat{\gamma}_{j}} \in W^{l+1 / 2}\left(\hat{\gamma}_{j}\right)$. Здесь $\langle\cdot, \cdot\rangle_{\hat{\gamma}_{j}},\langle\cdot, \cdot\rangle_{\gamma_{j}}-$ полуторалинейные формы на дуальных парах $W^{l+1 / 2}\left(\hat{\gamma}_{j}\right)$, $W^{-l-1 / 2}\left(\hat{\gamma}_{j}\right)$ и $E^{l+1 / 2}\left(\gamma_{j}\right), E^{-l-1 / 2}\left(\gamma_{j}\right)$ соответственно. Аналогично, для $g \in$ $\left(E_{a}^{l+1 / 2}(\gamma)\right)^{*}$ вводится распределение $\psi_{p} g_{j} \in W^{-l-1 / 2}(\hat{\gamma})$.

Для оператора $\mathscr{L}_{K}: E_{a}^{2}(K) \rightarrow E_{a}^{0}(K, \gamma)$, введенного в разделе 1 , рассмотрим сопряженньй оператор

$$
\mathscr{L}_{K}^{*}:\left(E_{a}^{0}(K, \gamma)\right)^{*} \rightarrow\left(E_{a}^{2}(K)\right)^{*}, \quad \text { где }\left(E_{a}^{0}(K, \gamma)\right)^{*}=E_{-a}^{0}(K) \times \prod_{j}\left(E_{a}^{3 / 2}\left(\gamma_{j}\right)\right)^{*} .
$$

Оператор $\mathscr{L}_{K}^{*}$ действует на $\left\{f, g_{j}\right\} \in\left(E_{a}^{0}(K, \gamma)\right)^{*}$ для любых $u \in E_{a}^{2}(K)$ по формуле

$$
\left\langle u, \mathscr{L}_{K}^{*}\left\{f, g_{j}\right\}\right\rangle=(\Delta u-u, f)_{K}+\sum_{j}\left\langle\left. u\right|_{\gamma_{j}}+\left.a_{j} u\left(\mathscr{G}_{j} y\right)\right|_{\gamma_{j}}, g_{j}\right\rangle_{\gamma_{j}} .
$$

Здесь $(\cdot, \cdot)_{K}$ обозначает скалярное произведение в $L_{2}(K)$, a $\langle\cdot, \cdot\rangle$ и $\langle\cdot, \cdot\rangle_{j}$ обозначают полуторалинейные формы на соответствующих дуальных парах пространств.

Для целых $l \geqslant 0$ положим $\mathscr{W}^{l}(K)=\bigoplus_{j} W^{l}\left(K_{j}\right)$.

Tеорема 4.3. Пусть $\left\{f, g_{j}\right\} \in\left(E_{a}^{0}(K, \gamma)\right)^{*}, \mathscr{L}_{K}^{*}\left\{f, g_{j}\right\} \in\left(E_{a}^{2}(K)\right)^{*}$. Тогда $n p u$ всяком иелом фиксированном $l \geqslant 0$ если

$$
\psi_{0} \mathscr{L}_{K}^{*}\left\{f, g_{j}\right\} \in\left\{\begin{array}{lll}
W_{\bar{K}}^{-2+l}\left(\mathbb{R}^{2}\right) & \text { npu } & l<2, \\
\mathscr{W}^{-2+l}(K) & \text { npu } & l \geqslant 2
\end{array}\right.
$$

$\operatorname{mo} \psi_{3}\left\{f, g_{j}\right\} \in \mathscr{W}^{l}(K) \times \prod_{j} W^{-3 / 2+l}\left(\hat{\gamma}_{j}\right) u$

$$
\begin{aligned}
& \left\|\psi_{3}\left\{f, g_{j}\right\}\right\|_{\mathscr{W}^{l}(K) \times \prod_{j} W^{-3 / 2+l}\left(\hat{\gamma}_{j}\right)} \\
& \quad \leqslant c_{l}\left(\left\|\psi_{0} \mathscr{L}_{K}^{*}\left\{f, g_{j}\right\}\right\|_{-2+l}+\left\|\psi_{0}\left\{f, g_{j}\right\}\right\|_{W_{\bar{K}}^{-1}\left(\mathbb{R}^{2}\right) \times \prod_{j} W^{-5 / 2}\left(\hat{\gamma}_{j}\right)}\right),
\end{aligned}
$$


əде

$$
\|\cdot\|_{-2+l}=\left\{\begin{array}{lll}
\|\cdot\|_{W_{\bar{K}}^{-2+l}\left(\mathbb{R}^{2}\right)} & \text { npu } & l<2, \\
\|\cdot\|_{\mathscr{W}-2+l}(K) & n p u & l \geqslant 2,
\end{array}\right.
$$

$c_{l}>0$ не зависит от $\left\{f, g_{j}\right\}$.

ДокАЗАТЕЛьство. 1) Введем оператор

$$
\mathscr{L}_{\mathscr{G}}^{*}: E_{-a}^{0}(K) \times \prod_{j}\left\{\left(E_{a}^{3 / 2}\left(\gamma_{j}\right)\right)^{*} \times\left(E_{a}^{3 / 2}(\gamma)\right)^{*}\right\} \rightarrow\left(E_{a}^{2}(K)\right)^{*},
$$

действующий на $\left\{f, g_{j}, g_{j}^{\prime}\right\} \in E_{-a}^{0}(K) \times \prod_{j}\left\{\left(E_{a}^{3 / 2}\left(\gamma_{j}\right)\right)^{*} \times\left(E_{a}^{3 / 2}(\gamma)\right)^{*}\right\}$ для любых $u \in E_{a}^{2}(K)$ по формуле

$$
\left\langle u, \mathscr{L}_{\mathscr{G}}^{*}\left\{f, g_{j}, g_{j}^{\prime}\right\}\right\rangle=(\Delta u-u, f)_{K}+\sum_{j}\left\{\left\langle\left. u\right|_{\gamma_{j}}, g_{j}\right\rangle_{\gamma_{j}}+\left\langle\left. a_{j} u\right|_{\gamma}, g_{j}^{\prime}\right\rangle_{\gamma}\right\} .
$$

Для $g_{j} \in\left(E_{a}^{3 / 2}\left(\gamma_{j}\right)\right)^{*}$ определим распределение $g_{j}^{\mathscr{G}} \in\left(E_{a}^{3 / 2}(\gamma)\right)^{*}$ соотношением

$$
\left\langle u_{\gamma}, g_{j}^{\mathscr{G}}\right\rangle_{\gamma}=\left\langle u_{\gamma}\left(\mathscr{G}_{j}\right), g_{j}\right\rangle_{\gamma_{j}} \quad \text { для всех } u_{\gamma} \in E_{a}^{3 / 2}(\gamma) \text {. }
$$

Заметим, что $\psi_{p} g_{j}^{\mathscr{G}} \in W^{-3 / 2+l}(\hat{\gamma})$ тогда и только тогда, когда $\psi_{p}\left(\mathscr{G}_{j}\right) g_{j} \in W^{-3 / 2+l}\left(\hat{\gamma}_{j}\right)$; при этом сушествуют константы $k_{1}, k_{2}>0$ (зависящие от $l$ ) такие, что

$$
k_{1}\left\|\psi_{p}\left(\mathscr{G}_{j} \cdot\right) g_{j}\right\|_{W^{-3 / 2+l}\left(\hat{\gamma}_{j}\right)} \leqslant\left\|\psi_{p} g_{j}^{\mathscr{G}}\right\|_{W^{-3 / 2+l}(\hat{\gamma})} \leqslant k_{2}\left\|\psi_{p}\left(\mathscr{G}_{j} \cdot\right) g_{j}\right\|_{W^{-3 / 2+l}\left(\hat{\gamma}_{j}\right)} .
$$

Из определения операторов $\mathscr{L}_{K}^{*}$ и $\mathscr{L}_{\mathscr{G}}^{*}$ следует, что

$$
\mathscr{L}_{\mathscr{G}}^{*}\left\{f, g_{j}, g_{j}^{\mathscr{G}}\right\}=\mathscr{L}_{K}^{*}\left\{f, g_{j}\right\} .
$$

2) Пусть $\varepsilon, \zeta, \zeta_{j}$ обозначают то же, что и в доказательстве леммы 3.1. Введем функции $\hat{\zeta}_{j}, \hat{\zeta}, \bar{\zeta}_{j}, \bar{\zeta} \in C_{0}^{\infty}(\mathbb{R})$ такие, что $\hat{\zeta}_{j}(\varphi)=1$ для $\left|b_{j}-\varphi\right|<3 \varepsilon / 2, \hat{\zeta}_{j}(\varphi)=0$ для $\left|b_{j}-\varphi\right|>2 \varepsilon ;$ $\hat{\zeta}(\varphi)=1$ для $|b-\varphi|<3 \varepsilon / 2, \hat{\zeta}(\varphi)=0$ для $|b-\varphi|>2 \varepsilon ; \bar{\zeta}_{j}(\varphi)=1$ для $\left|b_{j}-\varphi\right|<\varepsilon / 8$, $\bar{\zeta}_{j}(\varphi)=0$ для $\left|b_{j}-\varphi\right|>\varepsilon / 4 ; \bar{\zeta}(\varphi)=1$ для $|b-\varphi|<\varepsilon / 8, \bar{\zeta}(\varphi)=0$ для $|b-\varphi|>\varepsilon / 4$.

Носитель функции $\zeta_{i}$ не пересекается с $\gamma$ и $\gamma_{k}$ при $k \neq i$, поэтому $\psi_{p} \zeta_{i} g_{k}=0, \psi_{p} \zeta_{i} g_{j}^{\mathscr{G}}$ $=0 ;$ следовательно,

$$
\left\langle u, \mathscr{L}_{\mathscr{G}}^{*}\left(\psi_{p} \zeta_{i}\left\{f, g_{j}, g_{j}^{\mathscr{G}}\right\}\right)\right\rangle=\left(\psi_{p} \zeta_{i} \Delta u-\psi_{p} \zeta_{i} u, f\right)_{K}+\left\langle\left.\left(\psi_{p} \zeta_{i} u\right)\right|_{\gamma_{i}}, g_{i}\right\rangle_{\gamma_{i}} .
$$

Поскольку замена переменных типа поворота переводит оператор Лапласа в оператор Лапласа и сохраняет принадлежность функций соответствующим пространствам Соболева, мы можем воспользоваться теоремой $4.3[11, \text { гл. } 2]^{1}$. Отсюда, из соотношения (4.16) и формулы Лейбница следует, что

$$
\begin{gathered}
\psi_{1} \zeta_{i}\left\{f, g_{j}, g_{j}^{\mathscr{G}}\right\} \in W^{l}(K) \times \prod_{j}\left\{W^{-3 / 2+l}\left(\hat{\gamma}_{j}\right) \times W^{-3 / 2+l}(\hat{\gamma})\right\}, \\
\left\|\psi_{1} \zeta_{i}\left\{f, g_{j}, g_{j}^{\mathscr{G}}\right\}\right\|_{W^{l}(K) \times \prod_{j}\left\{W^{-3 / 2+l}\left(\hat{\gamma}_{j}\right) \times W^{-3 / 2+l}(\hat{\gamma})\right\}} \\
\leqslant k_{3}\left(\left\|\psi_{0} \mathscr{L}_{K}^{*}\left\{f, g_{j}\right\}\right\|_{-2+l}+\left\|\psi_{0} \hat{\zeta}_{i} f\right\|_{W_{K}^{-1}\left(\mathbb{R}^{2}\right)}+\left\|\psi_{0} g_{i}\right\|_{W^{-5 / 2}\left(\hat{\gamma}_{i}\right)}\right) .
\end{gathered}
$$

\footnotetext{
${ }^{1} \mathrm{~B}$ теореме 4.3 [11, гл. 2] рассматриваются операторы с переменными коэффициентами, из-за чего нак ладываются дополнительные ограничения наносители рассматриваемых функций. Однако легко видеть, что в случае операторов с постоянными коэффициентами эти ограничения могут быть сняты.
} 
Отсюда, в частности, при помощи (4.15) получим, что $\psi_{2} g_{i}^{\mathscr{G}} \in W^{-3 / 2+l}(\hat{\gamma})$ и $\left\|\psi_{2} g_{i}^{\mathscr{G}}\right\|_{W^{-3 / 2+l}(\hat{\gamma})} \leqslant k_{4}\left(\left\|\psi_{0} \mathscr{L}_{K}^{*}\left\{f, g_{j}\right\}\right\|_{-2+l}+\left\|\psi_{0} \hat{\zeta}_{i} f\right\|_{W_{\bar{K}}^{-1}\left(\mathbb{R}^{2}\right)}+\left\|\psi_{0} g_{i}\right\|_{W^{-5 / 2}\left(\hat{\gamma}_{i}\right)}\right)$.

3) Носитель функции $\zeta$ не пересекается с $\gamma_{j}$, поэтому $\psi_{p} \zeta_{i} g_{j}=0$; следовательно,

$$
\left\langle u, \mathscr{L}_{\mathscr{G}}^{*}\left(\psi_{p} \zeta\left\{f, g_{j}, g_{j}^{\mathscr{G}}\right\}\right)\right\rangle=\left(\psi_{p} \zeta \Delta u-\psi_{p} \zeta u, f\right)_{K}+\sum_{k}\left\langle\left.\left(\psi_{p} \zeta u\right)\right|_{\gamma}, g_{k}^{\mathscr{G}}\right\rangle_{\gamma}
$$

Таким образом, учитьвая свойства носителей функций $\psi_{p}$ и $\zeta$, оператор $\mathscr{L}_{\mathscr{G}}^{*}$, действующий на $\psi_{p} \zeta\left\{f, g_{j}, g_{j}^{\mathscr{G}}\right\}$, можно трактовать как сопряженный к оператору задачи

$$
\Delta u-u=\hat{f}(y), \quad y \in \mathbb{R}^{2},\left.\quad u\right|_{\hat{\gamma}}=\hat{g}_{1}(y),\left.\quad u\right|_{\hat{\gamma}}=\hat{g}_{2}(y), \quad y \in \hat{\gamma},
$$

которая (после соответствующей замены переменных типа поворота) совпадает с точностью до младшего члена $u$ с задачей п. 3 данного раздела. По доказанному вьше $\psi_{2} g_{k}^{\mathscr{G}} \in W^{-3 / 2+l}(\hat{\gamma})$; поэтому мы можем воспользоваться леммой 4.4. Тогда из соотношения (4.16) и формулы Лейбница получим

$$
\begin{gathered}
\psi_{3} \zeta\left\{f, g_{j}, g_{j}^{\mathscr{G}}\right\} \in \mathscr{W}^{l}(K) \times \prod_{j}\left\{W^{-3 / 2+l}\left(\hat{\gamma}_{j}\right) \times W^{-3 / 2+l}(\hat{\gamma})\right\}, \\
\left\|\psi_{3} \zeta\left\{f, g_{j}, g_{j}^{\mathscr{G}}\right\}\right\|_{\mathscr{W}^{l}(K) \times \prod_{j}\left\{W^{-3 / 2+l}\left(\hat{\gamma}_{j}\right) \times W^{-3 / 2+l}(\hat{\gamma})\right\}} \\
\leqslant k_{5}\left(\left\|\psi_{2} \mathscr{L}_{K}^{*}\left\{f, g_{j}\right\}\right\|_{-2+l}+\left\|\psi_{2} \hat{\zeta} f\right\|_{W_{\bar{K}}^{-1}\left(\mathbb{R}^{2}\right)}+\sum_{j}\left\|\psi_{2} g_{j}^{\mathscr{G}}\right\|_{W^{-3 / 2+l}(\hat{\gamma})}\right) .
\end{gathered}
$$

Отметим, что именно здесь возникает пространство $\mathscr{W}^{l}(K)$, т.е. гладкость функции $f$ нарушается на луче $\gamma$. Это происходит из-за наличия нелокальных членов в краевом условии (1.7) и, как следствие, в сопряженном операторе $\mathscr{L}_{K}^{*}$.

Из неравенств (4.19) и (4.18) имеем

$$
\begin{aligned}
& \left\|\psi_{3} \zeta\left\{f, g_{j}, g_{j}^{\mathscr{G}}\right\}\right\|_{\mathscr{W}^{l}(K) \times \prod_{j}\left\{W^{-3 / 2+l}\left(\hat{\gamma}_{j}\right) \times W^{-3 / 2+l}(\hat{\gamma})\right\}} \\
& \quad \leqslant k_{6}\left(\left\|\psi_{0} \mathscr{L}_{K}^{*}\left\{f, g_{j}\right\}\right\|_{-2+l}+\left\|\psi_{0} \hat{\zeta} f\right\|_{W_{\bar{K}}^{-1}\left(\mathbb{R}^{2}\right)}+\sum_{j}\left\|\psi_{0} g_{j}\right\|_{W^{-5 / 2}\left(\hat{\gamma}_{j}\right)}\right) .
\end{aligned}
$$

4) Носитель функции $\zeta_{0}=1-\sum_{i} \zeta_{i}-\zeta$ не пересекается с $\gamma_{j}$ и $\gamma$, поэтому $\psi_{p} \zeta_{0} g_{j}=0$, $\psi_{p} \zeta_{0} g_{j}^{\mathscr{G}}=0 ;$ следовательно,

$$
\left\langle u, \mathscr{L}_{\mathscr{G}}^{*}\left(\psi_{p} \zeta_{0}\left\{f, g_{j}, g_{j}^{\mathscr{G}}\right\}\right)\right\rangle=\left(\psi_{p} \zeta_{0} \Delta u-\psi_{p} \zeta_{0} u, f\right)_{K} .
$$

Из теоремы $3.1[11$, гл. 2], соотношения (4.16) и формулы Лейбница получим

$$
\begin{gathered}
\psi_{1} \zeta_{0}\left\{f, g_{j}, g_{j}^{\mathscr{G}}\right\} \in W^{l}(K) \times \prod_{j}\left\{W^{-3 / 2+l}\left(\hat{\gamma}_{j}\right) \times W^{-3 / 2+l}(\hat{\gamma})\right\} \\
\left\|\psi_{1} \zeta_{0}\left\{f, g_{j}, g_{j}^{\mathscr{G}}\right\}\right\|_{W^{l}(K) \times \prod_{j}\left\{W^{-3 / 2+l}\left(\hat{\gamma}_{j}\right) \times W^{-3 / 2+l}(\hat{\gamma})\right\}} \\
\leqslant k_{7}\left(\left\|\psi_{0} \mathscr{L}_{K}^{*}\left\{f, g_{j}\right\}\right\|_{-2+l}+\left\|\psi_{0} \bar{\zeta}_{0} f\right\|_{W_{K}^{-1}\left(\mathbb{R}^{2}\right)}\right)
\end{gathered}
$$

где $\bar{\zeta}_{0}=1-\sum_{i} \bar{\zeta}_{i}-\bar{\zeta}$.

Теперь априорная оценка (4.14) вытекает из неравенств (4.17), (4.20) и (4.21). 


\section{5. Разрешимость нелокальных эллиптических краевых задач}

В данном разделе собраны основные результаты о разрешимости нелокальных задач в плоских и двугранных углах (теоремы 5.1-5.3).

5.1. Прежде всего установим связь между ядрами операторов $\mathscr{L}_{K}^{*}$ и $\mathscr{M}_{K}$.

Лемма 5.1. Ядро $\operatorname{ker}\left(\mathscr{L}_{K}^{*}\right)$ оператора $\mathscr{L}_{K}^{*}$ совпадает с множеством, которое пробегает әлемент $\left\{v,\left.\left(\partial v_{j} / \partial n_{j}\right)\right|_{\gamma_{j}}\right\}$, когда $v \in \mathscr{E}_{-a+2}^{2}(K), v_{j} \in C^{\infty}\left(\bar{K}_{j} \backslash\{0\}\right) u$ удовлетворяет задаче $(3.1),(3.2) n p u\left\{f, g_{j}, h_{\nu}\right\}=0$. Здесь $v(y) \equiv v_{j}(y), f(y) \equiv f_{j}(y)$ при $y \in K_{j}$.

ДокаЗАТЕльСтво. 1) Пусть $v \in \mathscr{E}_{-a+2}^{2}(K), v_{j} \in C^{\infty}\left(\bar{K}_{j} \backslash\{0\}\right)$ и удовлетворяет задаче (3.1), (3.2) при $\left\{f, g_{j}, h_{\nu}\right\}=0$. Тогда для любой $u \in C_{0}^{\infty}\left(\bar{K}_{j} \backslash\{0\}\right)$ в силу теоремы 2.1

$$
\sum_{j=1}\left(\Delta u-u, v_{j}\right)_{K_{j}}+\sum_{j}\left(\left.u\right|_{\gamma_{j}}+\left.a_{j} u\left(\mathscr{G}_{j} y\right)\right|_{\gamma_{j}},\left.\frac{\partial v_{j}}{\partial n_{j}}\right|_{\gamma_{j}}\right)_{\gamma_{j}}=0
$$

Из непрерывности оператора вложения $\mathscr{E}_{-a+2}^{2}(K)$ в $E_{-a}^{0}(K)$ следует, что $v \in E_{-a}^{0}(K)$. Кроме того, по неравенству Коши-Буняковского и теореме 1.4

$$
\begin{aligned}
\left|\left(u_{\gamma_{j}},\left.\frac{\partial v_{j}}{\partial n_{j}}\right|_{\gamma_{j}}\right)_{\gamma_{j}}\right|^{2} & \leqslant\left. k_{1} \int_{\gamma_{j}} r^{2(a-3 / 2)}\left|u_{\gamma_{j}}\right|^{2} d \gamma \cdot \int_{\gamma_{j}} r^{2(-a+3 / 2)}\left|\frac{\partial v_{j}}{\partial n_{j}}\right|_{\gamma_{j}}\right|^{2} d \gamma \\
& \leqslant k_{2}\left\|u_{\gamma_{j}}\right\|_{E_{a}^{3 / 2}\left(\gamma_{j}\right)}^{2} \cdot\left\|\left.\frac{\partial v_{j}}{\partial n_{j}}\right|_{\gamma_{j}}\right\|_{E_{-a+2}^{1 / 2}\left(\gamma_{j}\right)}^{2}
\end{aligned}
$$

для всех $u_{\gamma_{j}} \in E_{a}^{3 / 2}\left(\gamma_{j}\right)$. Следовательно, $\left.\left(\partial v_{j} / \partial n_{j}\right)\right|_{\gamma_{j}} \in\left(E_{a}^{3 / 2}\left(\gamma_{j}\right)\right)^{*}$.

Таким образом,

$$
\left\{v,\left.\frac{\partial v_{j}}{\partial n_{j}}\right|_{\gamma_{j}}\right\} \in E_{-a}^{0}(K) \times \prod_{j}\left(E_{a}^{3 / 2}\left(\gamma_{j}\right)\right)^{*}
$$

и в силу определения оператора $\mathscr{L}_{K}^{*}$ и тождества (5.1)

$$
\left\langle u, \mathscr{L}_{K}^{*}\left\{v,\left.\frac{\partial v_{j}}{\partial n_{j}}\right|_{\gamma_{j}}\right\}\right\rangle=0 \quad \text { для всех } u \in C_{0}^{\infty}(\bar{K} \backslash\{0\}) .
$$

Но $C_{0}^{\infty}(\bar{K} \backslash\{0\})$ плотно в $E_{a}^{2}(K)$, следовательно, $\left\{v,\left.\left(\partial v_{j} / \partial n_{j}\right)\right|_{\gamma_{j}}\right\} \in \operatorname{ker}\left(\mathscr{L}_{K}^{*}\right)$.

2) Пусть теперь, наоборот, $\left\{v, \psi_{j}\right\} \in \operatorname{ker}\left(\mathscr{L}_{K}^{*}\right)$. Из теоремы 4.3 следует, что $v_{j} \in$ $C^{\infty}\left(\bar{K}_{j} \backslash\{0\}\right), \psi_{j} \in C^{\infty}\left(\gamma_{j}\right)$. Тогда из определения оператора $\mathscr{L}_{K}^{*}$ вытекает, что для любого $u \in C_{0}^{\infty}(\bar{K} \backslash\{0\})$

$$
(\Delta u-u, v)_{K}=-\sum_{j}\left(\left.u\right|_{\gamma_{j}}+\left.a_{j} u\left(\mathscr{G}_{j} y\right)\right|_{\gamma_{j}}, \psi_{j}\right)_{\gamma_{j}},
$$


что совместно с формулой Грина (2.1) дает

$$
\begin{aligned}
& \sum_{j}\left(\left.u\right|_{\gamma_{j}}+\left.a_{j} u\left(\mathscr{G}_{j} y\right)\right|_{\gamma_{j}},\left.\frac{\partial v_{j}}{\partial n_{j}}\right|_{\gamma_{j}}-\psi_{j}\right)_{\gamma_{j}} \\
& \quad+\left(\left.u\right|_{\gamma},\left.\frac{\partial v_{2}}{\partial n}\right|_{\gamma}-\left.\frac{\partial v_{1}}{\partial n}\right|_{\gamma}-\left.\sum_{k} \bar{a}_{k} \chi_{k}^{-1} \frac{\partial v_{k}}{\partial n_{k}}\left(\mathscr{G}_{k}^{-1} y\right)\right|_{\gamma}\right)_{\gamma} \\
& =\sum_{j}\left(u, \Delta v_{j}-v_{j}\right)_{K_{j}}+\sum_{j}\left(\left.\frac{\partial u}{\partial n_{j}}\right|_{\gamma_{j}},\left.v_{j}\right|_{\gamma_{j}}\right)_{\gamma_{j}}+\left(\left.\frac{\partial u}{\partial n}\right|_{\gamma},\left.v_{2}\right|_{\gamma}-\left.v_{1}\right|_{\gamma}\right)_{\gamma}
\end{aligned}
$$

Полагая $\operatorname{supp} u \in K_{j}$, из (5.2) получим, что $\Delta v_{j}-v_{j}=0$.

В силу леммы $2.2\left[11\right.$, гл. 2] для всякой системы функций $\left\{\Theta_{j \mu}\right\}_{\mu=1}^{2}$ из $C_{0}^{\infty}\left(\gamma_{j}\right)$ найдется функция $u \in C_{0}^{\infty}(\bar{K} \backslash\{0\})$ такая, что

$$
\left.u\right|_{\gamma_{j}}=\Theta_{j 1},\left.\quad \frac{\partial u}{\partial n_{j}}\right|_{\gamma_{j}}=\Theta_{j 2}, \quad u=0 \text { в окрестности } \gamma
$$

Отсюда, из (5.2) и того, что $\Delta v_{j}-v_{j}=0$, получим $\left.\left(\partial v_{j} / \partial n_{j}\right)\right|_{\gamma_{j}}-\psi_{j}=0$ и $\left.v_{j}\right|_{\gamma_{j}}=0$. Аналогично,

$$
\left.v_{2}\right|_{\gamma}-\left.v_{1}\right|_{\gamma}=0,\left.\quad \frac{\partial v_{2}}{\partial n}\right|_{\gamma}-\left.\frac{\partial v_{1}}{\partial n}\right|_{\gamma}-\left.\sum_{k} \bar{a}_{k} \chi_{k}^{-1} \frac{\partial v_{k}}{\partial n_{k}}\left(\mathscr{G}_{k}^{-1} y\right)\right|_{\gamma}=0
$$

Наконец, из того, что $v \in E_{-a}^{0}(K), v_{j} \in C^{\infty}\left(\bar{K}_{j} \backslash\{0\}\right)$, и теоремы 4.1 вытекает принадлежность функции $v$ пространству $\mathscr{E}_{-a+2}^{2}(K)$.

Теорема 5.1. Onератор $\mathscr{L}_{K}$ фредгольмов тогда и только тогда, когда на прямой $\operatorname{Im} \lambda=a-1$ нет полюсов оператор-функиии $\widetilde{\mathscr{L}}^{-1}(\lambda)$.

ДокАЗАТЕЛьСтво. Пусть оператор $\mathscr{L}_{K}$ фредгольмов, тогда по теореме $7.1[13]$ имеет место оценка (1.11) и, следовательно, по теореме 1.1 на прямой $\operatorname{Im} \lambda=a-1$ нет полюсов оператор-функции $\widetilde{\mathscr{L}}^{-1}(\lambda)$.

Пусть, наоборот, на прямой $\operatorname{Im} \lambda=a-1$ нет полюсов оператор-функции $\widetilde{\mathscr{L}}^{-1}(\lambda)$. Тогда по теореме 1.1 оператор $\mathscr{L}_{K}$ имеет конечномерное ядро и замкнутьй образ.

Докажем, что ядро оператора $\mathscr{L}_{K}^{*}$ конечномерно. Из формулы Грина (2.3), замечания 2.2 и лемм $1.5,3.2$ вытекает, что $\lambda_{0}$ является полюсом оператора $\tilde{\mathscr{L}}^{-1}(\lambda)$ тогда и только тогда, когда $\bar{\lambda}_{0}$ является полюсом оператора $\widetilde{\mathscr{M}}^{-1}(\lambda)$. Значит, на прямой $\operatorname{Im} \lambda=-a+1$ нет полюсов оператор-функции $\widetilde{\mathscr{M}}^{-1}(\lambda)$. Следовательно, по теореме 4.2 оператор $\mathscr{M}_{K}$ имеет конечномерное ядро, размерность которого в силу леммы 5.1 совпадает с размерностью ядра оператора $\mathscr{L}_{K}^{*}$.

5.2. Перейдем к изучению разрешимости нелокальной краевой задачи (1.1), (1.2) в двугранном угле. Сводя задачу (1.1), (1.2) к задаче (1.6), (1.7) с помощью преобразования Фурье по $z: U(y, z) \rightarrow \widehat{U}(y, \eta)$ и замены переменных $y^{\prime}=|\eta| \cdot y$, аналогично доказательству леммы 7.3 [6] получим следующий результат. 
TЕОрема 5.2. Пусть на прямой $\operatorname{Im} \lambda=a-1$ нет полюсов оператор-функиии $\widetilde{\mathscr{L}}^{-1}(\lambda) u \operatorname{dim} \operatorname{ker} \mathscr{L}_{K}=\operatorname{codim} \mathscr{R}\left(\mathscr{L}_{K}\right)=0$. Тогда оператор $\mathscr{L}_{\Omega}$ есть изоморфизм.

Используя формулу Грина для нелокальной задачи (1.1), (1.2) в двугранном угле $\Omega$, аналогичную формуле Грина (2.1) для задачи $(1.6),(1.7)$ в плоском угле $K$, и повторяя рассуждения работы $[6, \S 8]$, получим следующее необходимое условие фредгольмовости оператора $\mathscr{L}_{\Omega}$.

Теорема 5.3. Если оператор $\mathscr{L}_{\Omega}$ фредгольмов, то оператор $\mathscr{L}_{K}$ есть изоморфизм.

Из теорем 1.1, 5.2 и 5.3 следует, что если $\mathscr{L}_{\Omega}$ фредгольмов, то он изоморфизм.

Автор выражает глубокую признательность профессору А. Л. Скубачевскому за постоянное внимание к данной работе.

\section{СПИСОК ЦИТИРОВАННОЙ ЛИТЕРАТУРЫ}

[1] Бицадзе А. В. Об одном классе условно разрешимых нелокальных краевых задач для гармонических функций // Докл. АН СССР. 1985. Т. 280. №3. С. 521-524.

[2] Скубачевский А. Л. Эллиптические задачи с нелокальными условиями вблизи границы // Матем. сб. 1986. Т. 129(171). № 2. С. 279-302.

[3] Скубачевский А. Л. Модельные нелокальные задачи для эллиптических уравнений в двугранных углах // Дифференц. уравнения. 1990. Т. 26. №1. С. 120-131.

[4] Скубачевский А. Л. О методе срезающих функций в теории нелокальных задач // Дифференц. уравнения. 1991. Т. 27. №1. С. 128-139.

[5] Кондратьев В. А. Краевые задачи для эллиптических уравнений в областях с коническими или угловыми точками // Тр. ММО. 1967. Т. 16. С. 209-292.

[6] Мазья В.Г., Пламеневский Б.А. $L_{p}$-оценки решений эллиптических краевых задач в областях с ребрами // Тр. ММО. 1978. Т. 37. С. 49-93.

[7] Назаров С. А., Пламеневский Б. А. Эллиптические задачи в областях с кусочно гладкой границей. М.: Наука, 1991.

[8] Ройтберг Я. А., Шефтель З. Г. Формула Грина и теоремы о гомеоморфизмах для нелокальных эллиптических задач // Докл. АН СССР. 1971. Т. 201. № 5. С. 1059-1062.

[9] Ильин В.А., Моисеев Е. И. Априорная оценка решения задачи, сопряженной к нелокальной краевой задаче первого рода // Дифференц. уравнения. 1988. Т. 24. № 5. С. 795-804.

[10] Агранович М. С., Вишик М. И. Эллиптические задачи с параметром и параболические задачи общего вида // УМН. 1964. Т. 19. № 3. С. 53-161.

[11] Лионс ЖК.-Л., Мадженес Э. Неоднородные граничные задачи и их приложения. М.: Мир, 1971.

[12] Ладыженская О. А. Краевые задачи математической физики. М.: Наука, 1973.

[13] Крейн С. Г. Линейные уравнения в банаховом пространстве. М.: Наука, 1971.

[14] Блехер П. М. Об операторах, зависящих мероморффно от параметра // Вестн. МГУ. Сер. 1. Матем., мех. 1969. № 5. С. 30-36.

[15] Шефтель З. Г. Энергетические неравенства и общие граничные задачи для эллиптических уравнений с разрывными коэффициентами // Сиб. матем. ж. 1965. Т. 6. № 3. С. 636-668.

Московский государственный авиационный институт (технический университет)

E-mail : gurevichp@mtelecom.ru

Поступило 12.11.2001 